Article

\title{
Interdependent Healthcare Critical Infrastructure Analysis in a Spatiotemporal Environment: A Case Study
}

\author{
Nivedita Nukavarapu * and Surya Durbha \\ Centre of Studies in Resources Engineering, Indian Institute of Technology Bombay, Powai, Mumbai 400076, \\ India; sdurbha@iitb.ac.in \\ * Correspondence: nnivedita@iitb.ac.in
}

Received: 3 April 2020; Accepted: 16 May 2020; Published: 11 June 2020

\begin{abstract}
During an urban flooding scenario, Healthcare Critical Infrastructure (HCI) represents a critical and essential resource. As the flood levels rise and the existing HCI facilities struggle to keep up with the pace, the under-preparedness of most urban cities to address this challenge becomes evident. Due to the disruptions in the interdependent Critical Infrastructures (CI) network (i.e., water supply, communications, electricity, transportation, etc.), during an urban flooding event, the operations at the healthcare CI facilities are inevitably affected. Hence, there is a need to identify cascading CI failure scenarios to visualize the propagation of failure of one $\mathrm{CI}$ facility to another $\mathrm{CI}$, which can impact vast geographical areas. The goal of this work is to develop an interdependent HCI simulation model in a spatiotemporal environment to understand the dynamics in real-time and model the propagation of cascading $\mathrm{CI}$ failures in an interdependent $\mathrm{HCI}$ network. The model is developed based on a real-world cascading CI failure case study on an interdependent $\mathrm{HCI}$ network during the flood disaster event in December 2015 at Chennai, TamilNadu, India. The interdependencies between the CI networks are modeled by using the Stochastic Colored Petri Net (SCPN) based modeling approach. SCPN is used to model a real-word process that occurs in parallel or concurrently. Furthermore, a geographic information system-based interface is integrated with the simulation model, to visualize the dynamic behavior of the interdependent HCI SCPN simulation model in a spatiotemporal environment. Such a dynamic simulation model can assist the decision-makers and emergency responders to rapidly simulate 'what if' kind of scenarios and consequently respond rapidly.
\end{abstract}

Keywords: healthcare critical infrastructure; geovisualization; geographic information system; colored petri net

\section{Introduction}

Understanding and rapidly responding to flood events in urban cities (cities such as Mumbai, Chennai, Hyderabad, Bangalore, etc., in India) is important for supporting its economic growth and resilience. An increase in unplanned urban settlements in metropolitan cities is resulting in the change of drainage characteristics of natural drainage areas. Hence, these natural catchments are unable to manage the increased volume of floodwater and the rate of surface runoff due to the encroachment of wetlands, floodplains, and blockage of the drainage channels due to the disposal of solid and liquid wastes [1]. Obstruction of floodways, natural water bodies causes loss of natural flood storage leading to urban floods. Urban flooding can have severe implications on the interdependent critical infrastructures. Critical Infrastructure (CI) is defined as the group of assets and technological networks or systems such as electrical supply, water supply, transport, communication, finance, healthcare, and information technology, etc., which are vital for the functioning of the economy and society [2-5]. 
Critical infrastructure (CI) networks are not independent functioning assets; they are interdependent. For example, the functioning of a healthcare critical infrastructure network is dependent on the electrical supply CI, water supply CI, transport CI, and communication CI, etc. The $\mathrm{CI}$ networks are large scale distributed systems interconnected with each other based on spatial or operational interdependencies. The interdependent CI networks form a high degree of a complex network consisting of different nodes, edges, and sets $[2,3,5]$.

In an Interdependent CI network due to an event when a failure or disruption occurs in one of the CI network nodes, the failure propagates to the dependent CI node disrupting the subsequent second CI network such kind of domino effect type of failure is called as "cascading failure". Cascading failure arises due to a failure in one of the CI network components because of spatial or logical vulnerabilities. Disruption of one of the CI services due to failure in one of the CI components (CI facilities such as hospitals, electrical substations, etc.) can initiate cascading effects within an infrastructure or, in the worst case, cause failures in other infrastructures, possibly disrupting vital services, and affecting the security and performance of the interdependent CI services or systems [4,6]. As shown in Figure 1, an interdependent $\mathrm{CI}$ network is a set of different types of complex CI networks consisting of different sets of nodes, edges.

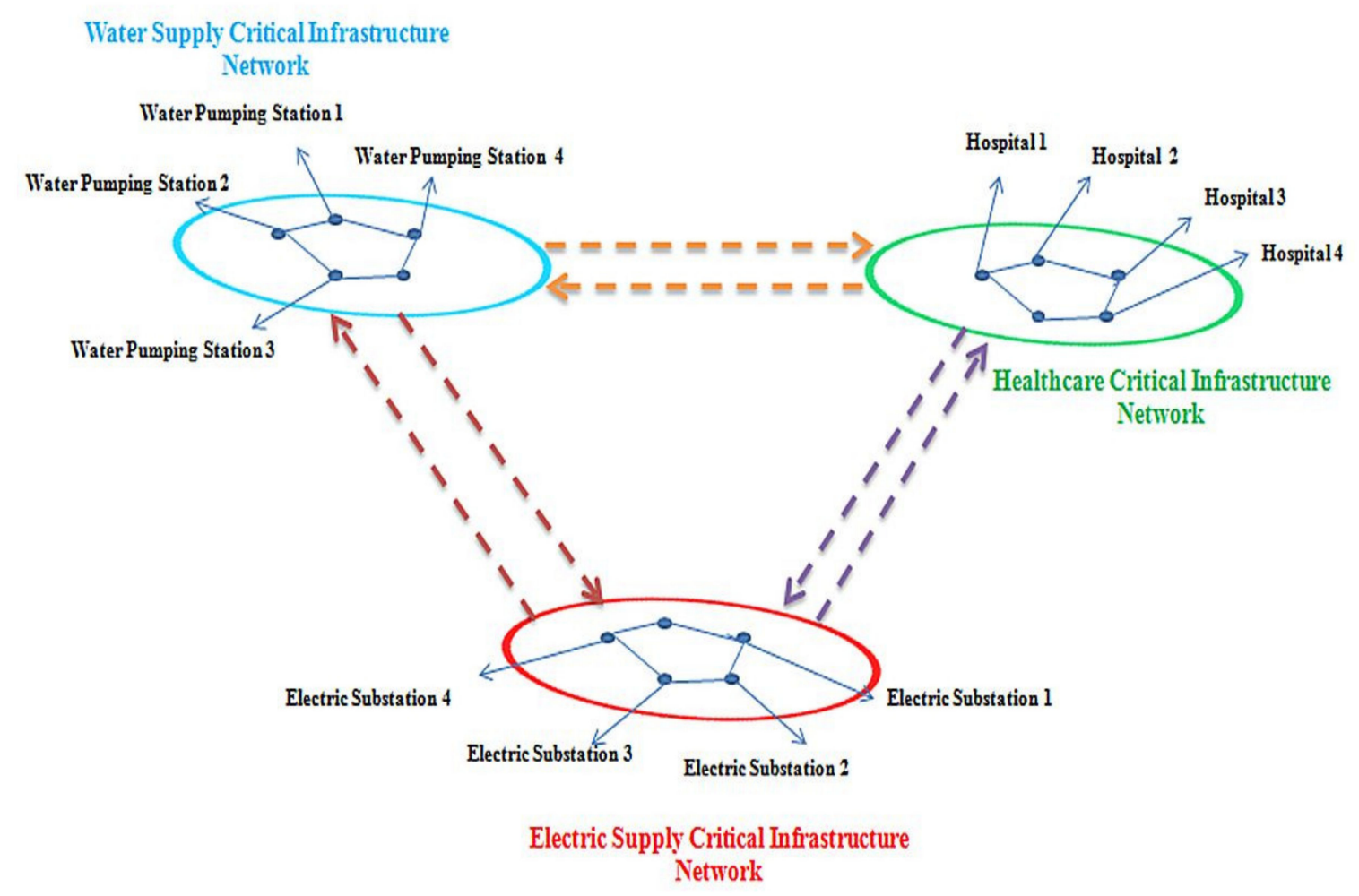

Figure 1. Interdependencies between the different Critical Infrastructure facilities nodes.

Each node in a CI network represents the CI facility. For example, in a healthcare CI network, each node represents the healthcare CI facility such as hospitals. In an electrical supply CI network, each node represents the electrical supply CI facility such as an electrical substation. The CI nodes are connected via links representing the relationship between them. In this complex interdependent network, the states of each CI node are correlated or are influenced by the state of the other CI node component. For example, the failed state of a CI facility node will disrupt the functioning of an interdependent operating CI facility node leading to a 'cascading failure' scenario. Such an event leaves the decision-makers and emergency personnel underprepared to deal with the impact of such cascading failure disruptions. 
During an urban flooding scenario, Healthcare Critical Infrastructure (HCI) represents a vital and crucial resource. As the flood levels rise and the existing HCI facilities fiercely struggle to keep up with the pace, their under-preparedness becomes evident. The healthcare operations are inevitably affected by disruptions in other critical infrastructures, and we certainly need to identify cascading scenarios and visualize the propagation of failure from one $\mathrm{CI}$ facility to another, which ultimately impacts the HCI facilities. In other words, the resilience of healthcare facilities cannot be treated in isolation but must be seen as part of a much bigger interdependent healthcare critical infrastructure (HCI) system $[7,8]$.

\subsection{Literature Review}

Extensive research has been done on the risk assessment of a single independent critical infrastructure facility. Although such analysis has importance in risk assessment, however interdependencies among the interlinked critical infrastructures should be considered for overall vulnerability assessment. Various approaches and techniques are being used to model critical infrastructure interdependencies, often based on the purpose of the outcome of the model, such as the empirical model, agent-based model, systems dynamic model, etc. The following literature showcases the different approaches to model critical infrastructure interdependencies. Wallace et al. $[9,10]$ modeled different infrastructure functionalities by using a uniform network flow mathematical representation. Chou and Tseng [11] proposed a knowledge discovery process, where they collected each CI facility failure history in the interdependent CI network and studied the failure patterns associated with their failure occurrence probabilities. Jha [12] developed a Bayesian Network (BN)-based model to analyze and predict the likelihood of a terrorist attack on the interdependent CI network. Barett et al. [13] Studied the interdependencies generated between the transportation $\mathrm{CI}$ and the communication CI due to human behavior at the time of crisis. Duenas-Osorio and Kwasinski [14] analyzed the interdependencies between the CI networks using a time series method from the post-disaster event utility restoration curves after the Chilean earthquake in 2010. Cardellini et al. [15] Used the agent-based approach to understanding the interdependencies between various interdependent $\mathrm{CI}$ in a single framework.

For simulation and modeling of the interdependencies between the healthcare critical infrastructure networks so far, the numbers of published studies are less. Loosemore et al. [8] analyzed the impact of an extreme event on an $\mathrm{HCI}$ facility and analyzed the interdependencies using rich picture diagrams. Arboleda et al. [16] developed network flow models designed to assess the impact of the loss of capability in the utility infrastructure networks on the ability of hospitals to provide adequate care for patients. Arboleda et al. [17] represented the interdependencies between different healthcare facilities and internal dependencies using a network flow model. John [18] developed an agent-based healthcare impact simulation model HCSim. The simulation model is used to assess the impact of a mass casualty event on the HCI facilities. Vugrin et al. [19] suggested that healthcare facilities need to absorb and adapt to disruptive circumstances in several ways, for example, making the structures more flood-resistant or ensuring sufficient physical capacity to absorb patients in a mass casualty event. The impact of a disaster event such as flooding on the internal and external interdependencies of a healthcare facility has been rarely addressed in the literature.

Petri Nets for Critical Infrastructure Interdependency Modeling

Petri net is a graph theory-based mathematical tool and is used to model complex network processes and analyze their performance and behavior [20,21]. Petri nets are useful to diagrammatically model concurrency and synchronization in distributed systems. Unlike the state machines, the state transitions in Petri nets are asynchronous. Various types of dynamic event-driven systems have been modeled by using Petri net, as in the real world system, events happen at the same time and the system may have many local states to form a global state. 
A Petri net enables a quick and intuitive representation of the different states, events, and interdependencies between the critical infrastructure systems. The probabilistic and temporal analysis provided by the Petri net is useful in understanding the behavior of the complex system. It offers a promising approach towards the risk modeling and analysis of interdependencies of critical infrastructures. Petri net approach is modular and scalable; as it is possible to add several new CI facilities, interdependencies, activities, or events that one likes to add to an existing system. The modeling approach provides the modeler to add more details, which means that we can model a complex system at a finer level of abstraction, by implementing nets within nets (which are called as subpages in the Petri net model) [22,23]. Petri nets are explained in detail in Section 2.1. Petri net in its various extensions such as colored Petri net has been used by researchers to capture and model the interdependency among the critical infrastructures [24-28].

Gursesli et al. [25] used the Petri net to develop a model the interdependencies among various CI such as electrical supply, transportation, water supply, communication, education and, emergency services. Sultana and Chen [26] studied and analyzed the interdependencies between the elements of the infrastructure used to generate hydroelectricity such as a dam, turbine, pumping station, etc., at the onset of a flood. The interdependencies were modeled by using an extended Petri net model and the behavior was analyzed by using Markov chain analysis. Krings et al. [27] developed a generalized stochastic Petri net (GSPN) for modeling the cascading failures in an interdependent CI network. Immediate and timed transitions were used to identify a common-mode fault. The interdependencies were modeled based on a scenario where the failure of the electrical supply CI would impact the other interdependent CI. Chen et al. [28] modeled cyber-physical attacks on the smart grid using Petri Net. Laprie et al. [29] provided a Petri-net model to study the interdependencies between electric supply CI and information technology. Omidvar et al. [30] Developed a Petri net model to understand and assess the failure risk between the various CI facilities in an interdependent CI network when an earthquake occurs. Giglio et al. [31] Proposed the Petri net simulation model to be used to analyze, control, and optimize a railway traffic system. Di Febbraro et al. [32] modeled the CI interdependencies and criticality of failure events in a railway network and analyzed the vulnerabilities of the network. Szpyrka et al. [33] modeled and evaluated the risk propagation of cybersecurity with Petri nets. Petri net simulation has been widely used in the healthcare domain. Dotoli et al. [34] proposed a timed Petri net model to simulate the workflow for a hospital department. Work by Hamana et al. [35] addressed the performance evaluation and verification of a healthcare facility and its services to a specific region or territory.

The versatility of the Petri simulation means that it can be used in a wide variety of applications. Most of the work done considered the workflow of a healthcare facility or the simulation of the services provided by a healthcare facility. Our work focuses on stochastic colored Petri net-based modeling and simulating of critical interdependencies in an interdependent healthcare critical infrastructure network during a flooding disaster event.

\subsection{Core Objectives}

In the last five years, many urban cities in India have witnessed a stream of extreme weather patterns that have left an unmistakable path of destruction. Experiences from the Mumbai floods 2005, Kashmir floods 2014, Chennai Floods 2015, and Kerala floods 2018 in India, has shown that urban flood disasters disrupt the operations of various CI networks and services; healthcare critical infrastructure (HCI) being one of the critical and vital among them.

During an urban flooding disaster scenario, the HCI network is called upon to provide emergency services. The HCI facilities must be able to provide the flood victims and patients in the hospital with medical care, pharmaceutical supplies, pathological services, intensive care services, etc., for an extended duration and under often difficult circumstances [7]. HCI facilities are at risk of damage from internal or external sources generated by an urban flood. Due to the disruption of lifeline services such as electricity supply, water supply, etc., the patients treated at the impacted HCI facility have to be 
transferred to another HCI facility. Hence, there is a need to model and simulate the system dynamics of an interdependent HCI network.

Given below is a real-life What-if Scenario showcasing the criticality of interdependencies between HCI facility networks during a flooding scenario, and the need for a simulation model for the decision-makers.

"A healthcare facility is flooded. The electrical substation which provides electric supply to the hospital is inundated. Due to which the electric supply to the healthcare facility (hospital) and the water pumping facility (water pumping station) is disrupted. Due to water pumping station disruption, the hospital is cut off from water supply. At the same time, as a result of the increase in flood water level, the basement of the hospital is inundated, and the backup generators became non-operational. The loss of electrical supply, water supply, and the unpreparedness of the hospital resulted in the operational failure of the hospital. The most affected area is the intensive care unit (ICU) of the hospital, due to non-functioning medical equipment and an insufficient number of reserved oxygen cylinders for the ICU patients. The ICU patients have to be evacuated in ambulances to the nearby safer hospital. The ambulances have to transport the patients via the shortest and the safest routes".

The end-user can query the simulation model for various flooding situation-based queries such as:

- Which CI facilities are inundated?

- What is the shortest and safest route for emergency evacuation of patients from the HCI facility?

- Which roads inundated?

- How much time is left for water supply disruption?

- What would be the cascading impact on the HCI facility if there is a failure in one of its interdependent CI facilities such as disruption in an electrical CI substation?

From the above scenario, we observe that the interdependent $\mathrm{HCI}$ network is at its vulnerable most during a flood event, and a real-time situational simulation model will help address these vulnerabilities. There is a dire need for an interdependent HCI network dynamic simulation model during a flood event, in a spatiotemporal environment. The model should be based on real-world scenarios, which exhibit concurrency, synchronization, and randomness. Both internal and external stochastic dependencies of the HCI network have to be addressed in the simulation model.

The core objectives of this work are:

To develop a stochastic colored Petri net driven geographic information system-based simulation model, useful in understanding the status of healthcare critical infrastructures during a flooding disaster scenario. This involves understanding the impact of different flood levels on the CI facilities using probabilistic fragility curves and the complex stochastic interdependencies between the CIs. The stochastic colored Petri net model is developed with various object-oriented functions and geospatial relationships, such that the Geographic Information System (GIS) is driven by the stochastic colored Petri net model. The coupling of the Petri net and the geographic information provides the simulation model with the spatiotemporal dimension.

The entire complex dynamic simulation model is then encapsulated into a java based Geovisualization interface. The utility of the approach is evaluated and analyzed using a real-world cascading CI failure case study scenario. The case study is based on an interdependent HCI network during the flood disaster event in December 2015 at Chennai, TamilNadu, India. The ability to have a stochastic colored Petri net driven geographic information system based interdependent critical infrastructure simulation model can have an impact on real-time decision-making heuristics during a flood disaster event.

The remaining paper is organized as follows; Section 2 discusses the Methodology. Section 3 consists of the development of the Stochastic Colored Petri net (SCPN) simulation model, in a geographical environment based on the Chennai 2015 flood disaster event case study. While Section 4 presents the results, where the model predicts the propagation of cascading failure scenarios and 
analyzes the system dynamics of an interdependent HCI network simulation model during the Chennai 2015 flood disaster event. Conclusions are drawn in Section 5.

\section{Methodology}

This section describes the methodology, which consists of detailed explanation of Petri net, its variation stochastic Petri net in detail, SCPN based interdependent healthcare CI simulation model system in a spatiotemporal environment, the proposed architectural framework of the system; probabilistic assessment for interdependent healthcare critical infrastructure network and the development of an SCPN driven geographic information system based interdependent healthcare critical infrastructure simulation model.

\subsection{Petri Nets}

Petri net is a directed, bipartite graph as shown in Figure 2; it is a powerful mathematical tool to model a complex process, as it provides a way to disintegrate the different states of a system as mentioned earlier in Section 1.1. It is defined as a five-tuple ( $P, T, I, M, O)$. Where ' $P$ ' denotes the set of places nodes (conditions of the network). ' $\mathrm{T}$ ' stands for transitions nodes (events in the network). ' $\mathrm{I}$ ' stand for input functions, assigned on the directed arcs, which connect the place node to a transition node (input arc) and ' $O$ ' stands for output functions, assigned on the directed arcs which connect the transition node to a place node (output arc) in a Petri net. Furthermore, tokens are assigned in a place which is called network 'marking' indicating the existing condition of the network. Each distinct marking in a Petri net represents a separate state of the Petri Net. An initial distribution of the tokens in the place nodes of a Petri net is called an initial marking [20,21,36].

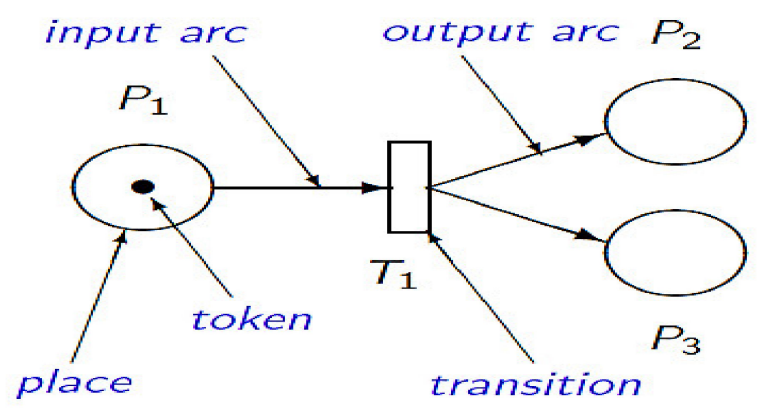

Figure 2. Basic Petri net.

- $\quad P=\{P 1, P 2, P 3 \ldots P n\}$ is a set of places in the Petri Net.

- $T=\{T 1, T 2, T 3 \ldots T n\}$ is the limited sets of transitions, $P \cup T \neq \emptyset, P \cap T \neq \emptyset$.

- $I: P \times T \rightarrow N$ denotes the set of Input directional arcs from place to transition, where $N=\{0,1,2 \ldots\}$;

- $O: P \times T \rightarrow N$ denotes the set of output directional arcs from Transition to places.

- $\quad M: P \rightarrow N$ denotes the marking of the Petri net, which defines the number of tokens present in the places, $M 0$ is the initial Marking.

An initial distribution of the tokens in the place nodes of a Petri-net is called initial marking. Once the transition in the Petri net is enabled at the onset of a simulation run, the token is being fired and removed from one place node (input) to another place node (output) by the transition. There needs to be at least one enabled transition for the firing of tokens to continue or else the execution gets stopped [21,37]. In respect to critical infrastructure interdependencies modeling, the place nodes with the token markings, in the Petri net simulation model, represent the states or conditions of the CI system or their components. The transitions represent the impacts across critical infrastructure networks or their components. The $\mathrm{CI}$ interdependencies are stimulated by the flow of the tokens throughout the network. 
Most of the studies that are present in the literature present us with Petri net simulation models that showcase the interdependencies between few CI nodes, in a small CI network. These Petri nets models cannot be used to model simulate and analyze the dynamics of the uncertainty of a large complex system. Hence, it is not possible by such Petri net models to simulate the real-life events which unfold during a flood event, which are discrete and stochastic. This limitation is addressed by developing a simulation model using Stochastic Colored Petri Net (SCPN), which increases the strength of the Petri Net and makes it possible to simulate a large real-time complex process [38,39].

The Colored Petri Nets (CPNs) $[38,39]$ formalism is an extension of the Petri net that is particularly suited to modeling and analyzing complex interconnected systems. In Colored Petri Net, the token present in a place node is distinguished by a color, where color represents the datatype or type (such as an integer, float, string, etc.). In particular, colors can be used to model different interactions between components or transect interdependencies between them. Moreover, CPNs combines the capabilities of Petri net with those of the Standard ML programming language [39,40]. The resulting flexibility and computational power make them a suitable formalism for modeling the complex interdependencies in the critical infrastructure network. More precisely since the notion of time is essential in the problem under study, we use a timed extension of CPNs, namely, Timed Colored Petri Nets (TCPNs). Timed Colored Petri nets allow adding timing information to CPN models; this is done by associating the transitions in the CPN model with token firing times. Depending on the firing times, a Petri net simulation model can be further divided as being deterministic or stochastic [24,39,41,42]. Thus, to model the stochastic interdependencies between the Critical Infrastructures, time-critical events, and cascading effects for a real-world complex system, a timed colored Petri net is the most suitable framework

\section{Stochastic Colored Petri Nets}

Stochastic Colored Petri nets (SCPN) contain timed transitions, which are associated with a firing rate that specifies a parameter of the exponential distribution of the time period between subsequent firings of the transition. Thus, SCPN can be used to model the timing behavior of an interdependent HCI network system. The firing rates of the tokens in the SCPN are proportional to the failure probabilities of the various components of the interdependent HCI network. In Timed Stochastic Colored Petri-Net (TSCPN), a transition requires a period of delay time from occurrence-able to occurrence of an event. In this context, the duration that a transition ' $t$ ' changes from the occurrence-able state to the occurrence state can be viewed as a stochastic variable ' $x i$ '. The stochastic variable ' $x i$ ' represents the delay time consumed by a certain CI for getting affected and damaged [42,43]. A detailed explanation of a TSCPN model is given in Figure 3.

The cascading failure of the critical infrastructure nodes at various time instants forms a stochastic process. To model the stochastic interdependencies between $\mathrm{HCI}$ and the other $\mathrm{CI}$, we need to identify the dependencies between the different variables. Such that when the process enters a particular state, it remains there for some period of (exponentially distributed) time and then moves to the next state.

To identify cascading effects and model the risk occurrence, the various parameters that need to be considered are; Probability of occurrence of the risk, the relationship between the risks and the different $\mathrm{CI}$ states of the interdependent $\mathrm{HCI}$ network and the time duration of the state of CI node when the risk will be in effect [7,39].

System analysis of Petri nets can be done using either analytical techniques such as Markov chain or simulation. Analytical techniques construct the so-called reachability graph from an SCPN, which is a labeled transition system, where each state represents a possible marking of the net. For large complex systems system analysis using analytical models such as Markov chains becomes impossible due to the state explosion problem [40]. 


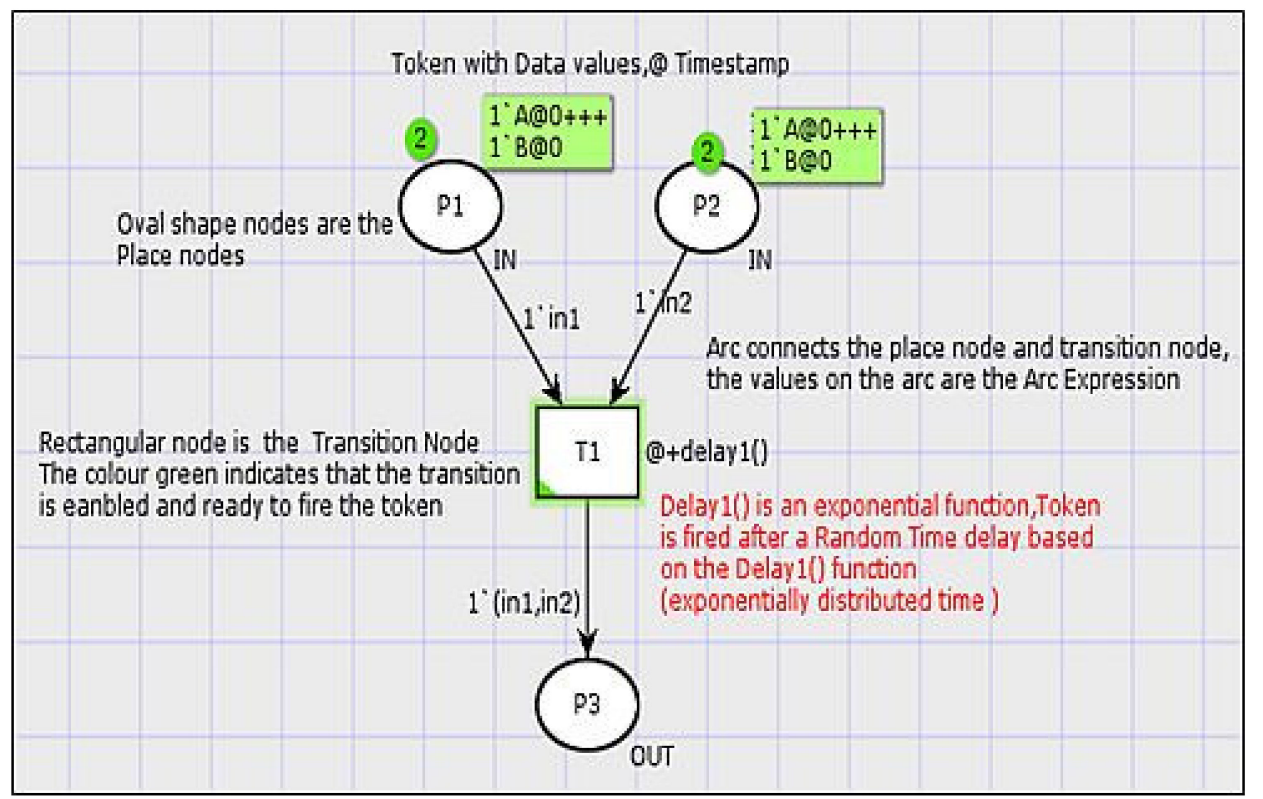

Figure 3. Example of a Timed Stochastic Colored Petri Net.

Instead, the system analysis can be performed from the quantitative data from the simulation data of SCPNs, after a simulation run reaches predefined stop criteria. As the simulation proceeds, the simulation model enters into different states. The state-space analysis provides knowledge about the various states of the SCPN simulation model. The data is extracted from the different states of the model and the events that occur during the simulation with the help of data collectors and data monitors [39]. The semantics of a Colored stochastic Petri net is equivalent to a Continuous Time Markov Chain (CTMC). The CTMC is constructed from the state space of the underlying SCPN by marking the arcs between states with the state transition rates. The system analysis component depicts the behavior of the system on its multiple simulation runs $[7,39,40]$. System analysis for the model under study is explained in detail in Section 4.1.

\subsection{End to End system of SCPN based Interdependent HCI Simulation Model System in a Spatiotemporal Environment}

The interdependencies between the various CI nodes are based on the logical, geographical, and physical relationship between them. The proposed end to end SCPN based interdependent HCI simulation model system in a spatiotemporal environment is presented in Figure 4 (more details in Section 3).

The direct impact of an individual Critical Infrastructure (CI) facility in a flooding event scenario is evaluated by the damage probabilities and analyzing the fragility curves. The damage probability of the flooding event for an infrastructure facility is defined based on the fragility curve (explained in Section 2.4). The failure probability of each critical infrastructure depends on the type and intensity of the flooding event while considering the local terrain and infrastructure structural characteristics. The stochastic colored Petri net simulation model presents the end-user with flood level simulation, the impact of flood level on each critical infrastructure facility, and the stochastic interdependencies between the HCI networks. Also, the simulation model predicts temporally, when each of the CI facilities will reach a non-operational state due to an increase in the flood level.

The dynamics and the propagation of the cascading failures between the various complex interdependencies between critical infrastructures related to healthcare infrastructure are visualized, in a spatiotemporal environment. The breakdown of each critical infrastructure is monitored, and the simulation model predicts the shutdown or collapse of the entire interdependent HCI network. The simulation model captures each state of the process; the state-space helps in analyzing the dynamics 
and the system analysis of the process. Modeling of the external dependencies related to a healthcare critical infrastructure is highly complex. However, the developed simulation model considers both the external and internal dependencies of a health care facility during a flood event.

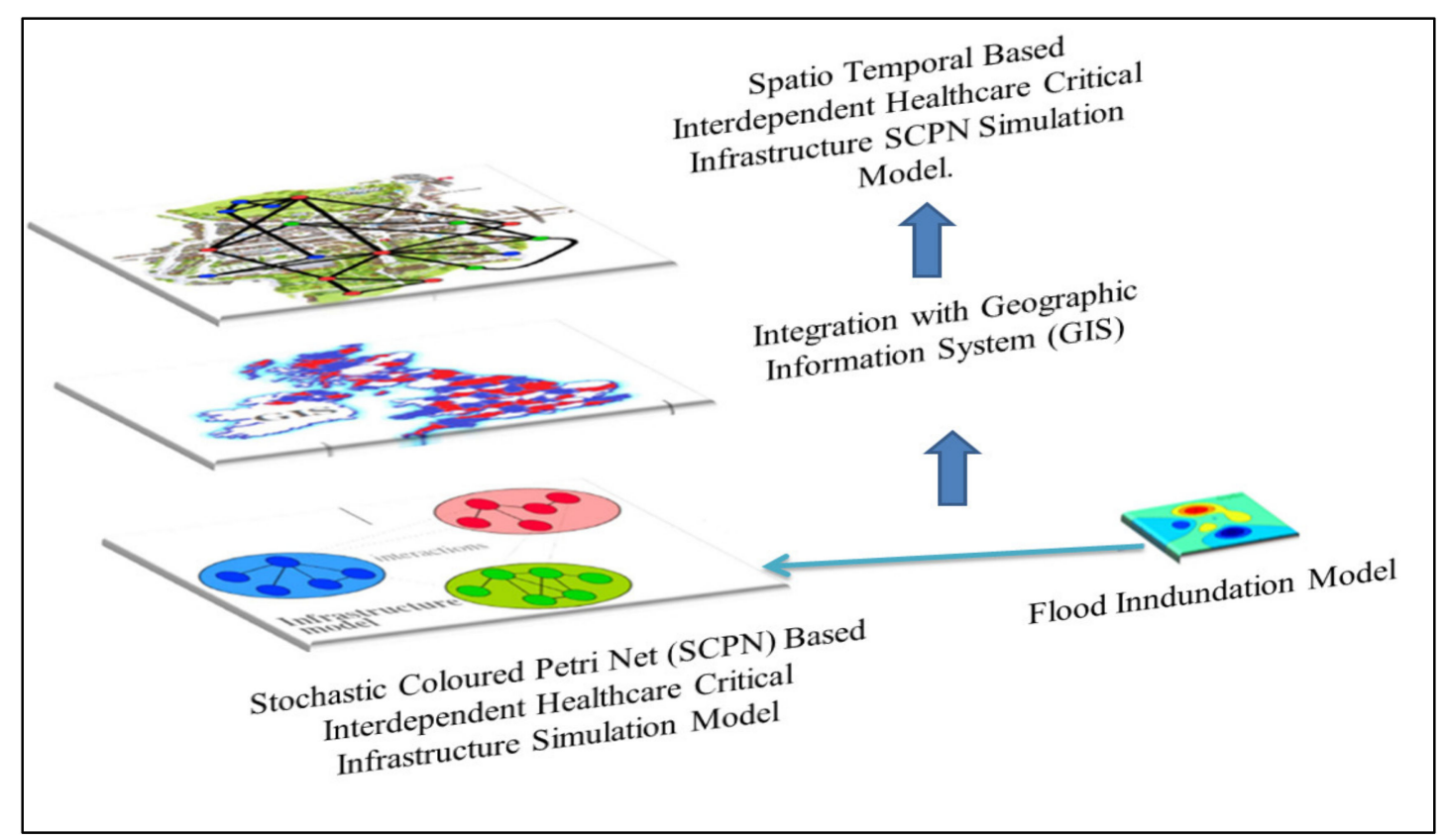

Figure 4. End to End Stochastic Colored Petri Net (SCPN) based interdependent Healthcare Critical Infrastructure (HCI) simulation model system in a spatiotemporal environment.

\subsection{Architectural Framework of the SCPN based Interdependent HCI Simulation Model}

The proposed architectural framework for spatiotemporal based interdependent healthcare critical infrastructure SCPN simulation model during a disaster is shown in Figure 5. The architecture of the entire system is shown in a bottom to top strategy approach of information processing. Based on this architectural framework the SCPN based interdependent HCI simulation model for the Chennai case study has been developed, which is discussed in detail in Sections 3 and 4. The various components of the proposed architecture have been explained in detail in the following sections.

(a) Data Level:

The data level of the architectural framework consists of different spatial geodatabases of various CI networks such as healthcare CI, electrical substation CI, etc., are associated with the CI facility nodes. The feature nodes of a CI facility are defined by their spatial attributes. For example, a healthcare CI geodatabase would have information such as Healthcare Facility (HF) geographic location, healthcare facility name, type of healthcare facility (Govt hospital, private hospital, poly-clinic, etc.), Intensive Care Unit (ICU) services, capacity (hospital beds) and ambulance services, etc.

(b) Middleware:

The stochastic interdependencies between the HCI networks are modeled using a stochastic Colored Petri net. The place nodes in the CSPN simulation model are linked with each other based on different geospatial interdependency rules between the various CI facilities in the HCI network. Geospatial relationship functions are used to connect the Petri net with the CI geographical features. The behavior and the system analysis are derived from its state-space analysis. Where all possible reachable states and state changes of a system model, are been identified and visualized. The CSPN simulation model is explained in detail in Section 3.

(c) Simulation Environment:

The SCPN driven GIS-based interdependent HCI simulation model is encapsulated in java based Geovisualization client interface. The client interface is enhanced with various visualization features 
and tools. The client interface also consists of a log window that keeps on updating the various statuses of the CI facilities. Which are if they are operating, inundation state, or a complete failure state.

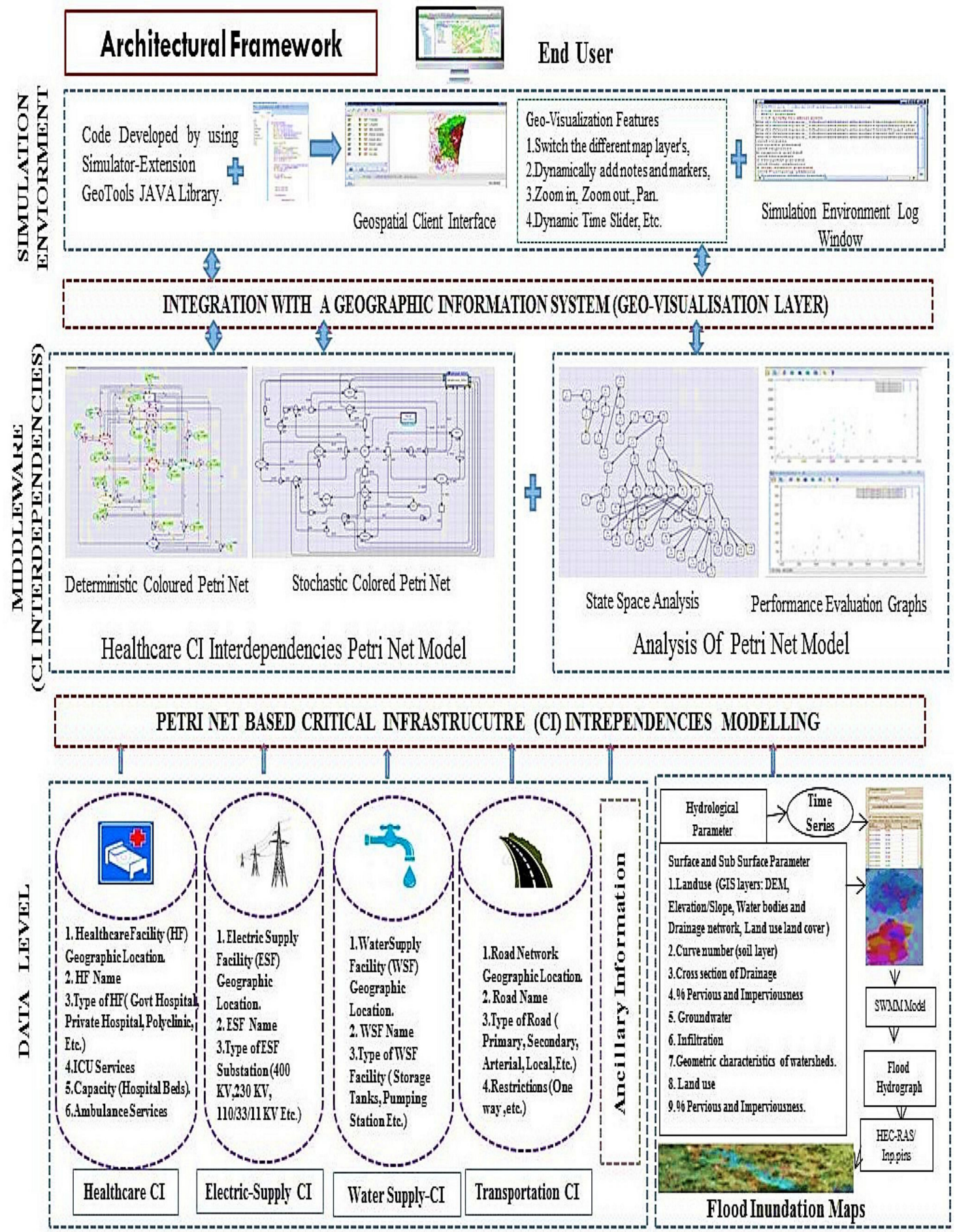

Figure 5. The proposed architectural framework for colored Petri net modeling for healthcare interdependencies during a flood event.

Temporal analysis of the HCI simulation model, as shown in Figure 6, enables us to examine and model the behavior of the interdependent healthcare $\mathrm{CI}$ network over time. For example, to determine whether and how the CI nodes are performing, under increasing flood levels over the time period. 
For an epoch unit of time, as the states of the various nodes change in the HCI simulation model, the visualization on the interdependent HCI network for an urban flood map changes. So for each simulation run and every state change, a new map is generated. The dynamic maps help us to analyze the criticality of the situation.

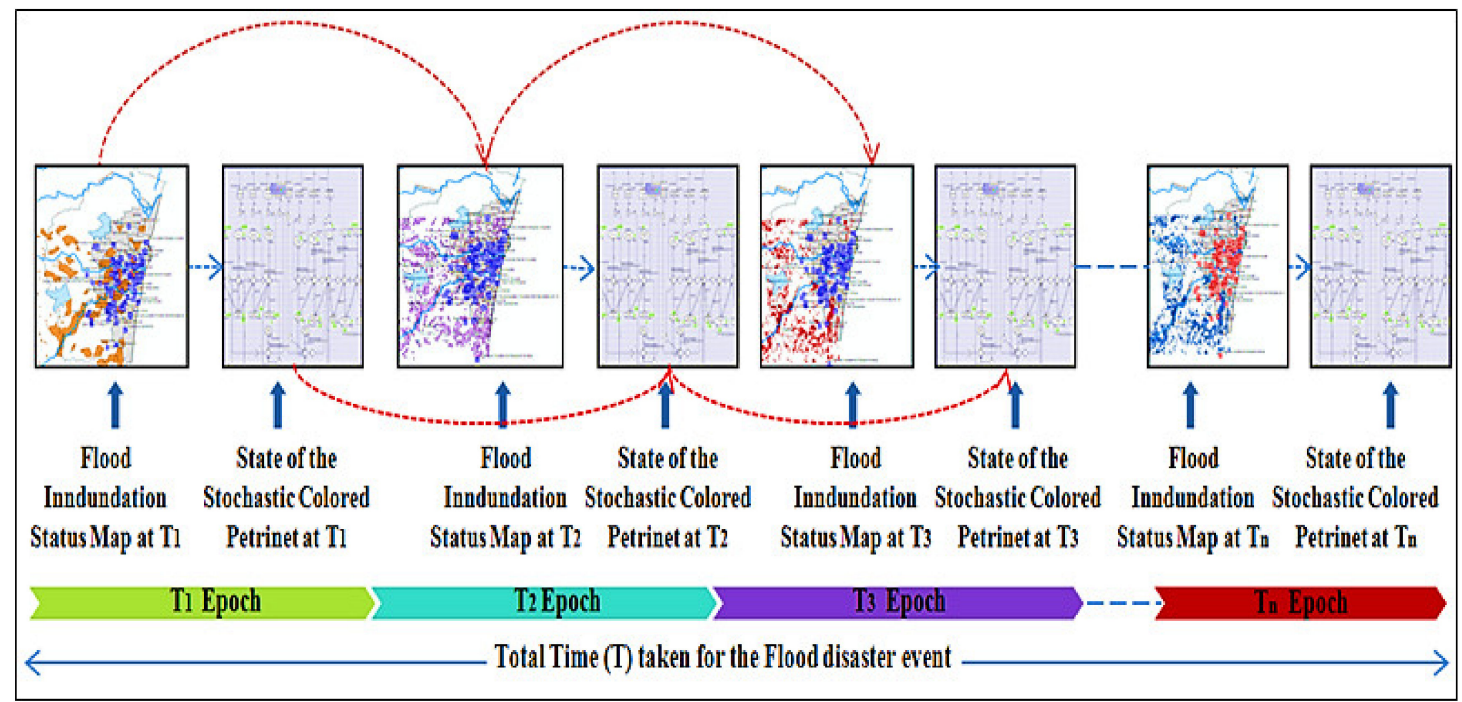

Figure 6. Temporal analysis of the SCPN interdependent HCI simulation model.

\subsection{Probabilistic Assessment for Interdependent Healthcare Critical Infrastructure (HCI) Network}

In a stochastic Colored Petri-net, a transition requires a period of delay time for an event to occur. That is the time duration required for a transition ' $t_{i}$ ' to change from state ' $\mathrm{S}_{1}$ ' to state ' $\mathrm{S}_{2}$ '. The time duration can be viewed as a stochastic variable ' $x_{\mathrm{i}}$ '. The stochastic variable ' $x_{\mathrm{i}}$ ' represents the delay time consumed by a certain CI for getting affected and damaged. As the firing times of the transition in the Petri net are stochastic, then the probability with which the transition ' $t_{i}$ ' will fire by time ' $x_{i}$ ' should be computed based on the distribution function of each transition as following:

$$
f\left(X_{i}\right)=\int_{0}^{X_{i}} f(x) d x
$$

where $f(x)$ is the probability density function of the firing for transition ' $t_{i}$ '. For example, if the failure rate of a CI facility for a flood event corresponding to a transition ' $t_{i}$ ' follows an exponential distribution, then the probability that transition ' $t_{i}$ ' will fire by the time ' $x$ ' is:

$$
f\left(X_{i}\right)=\int_{0}^{X_{i}} \lambda_{i} e^{-\lambda_{i} x} d x
$$

As the simulation model is based on an urban flood scenario, the CI nodes would reach a failed state due to increasing flood levels. Once the $\mathrm{CI}$ unit reaches a failed state, it would remain in that state until a repair action is initiated to restore the $\mathrm{CI}$ node to a working condition. During an urban flood scenario, the repair time of the CI node takes quite some time. As foremost, the flooding has to be controlled, so we are not considering the time taken to repair the CI node.

However, as we are simulating the domino effect of the interdependent $\mathrm{CI}$ network from the onset of the flood, we are interested in the time to failure component of the CI node. That is the instant at which the CI node experiences failure. These time instants are random variables, linked to the probability of an event. 
Let us consider a CI node facility (electrical substation ' $A$ ') from the electrical supply critical infrastructure network. Considering the electrical substation at time $t=0$ is in operating condition, the instant at a time at which it will eventually fail is a continuous random variable $X \geq 0$. Where ' $X$ ' represents the time to failure of Electrical substation $A$. ' $X$ ' is a random variable, and its range is the set of all positive real numbers, including zero $\left(X \in R^{+} \cup\{0\}\right)[22,24,44]$.

The cumulative distribution function $(\mathrm{CDF}) F_{X}(t)$ of the time to failure ' $\mathrm{X}$ ' is defined as

$$
F_{X}(t)=P\{X \leq t\}
$$

The probability density function (PDF) of the random variable ' $\mathrm{X}$ ' is given by

$$
f_{X}(t)=\frac{d F_{X}(t)}{d t}
$$

The hazard rate of a random variable ' $X$ ' is defined as the conditional probability that ' $X$ ' takes a value in the interval $(t, t+d t)$, given that $X>t$. Where ' $X$ ' is the time to failure of a unit, the hazard rate is called as the failure rate and is denoted by ' $\lambda$ '. The failure rate of a CI facility node gives us the probability that the failure will occur in the next unit of time $[31,33,35]$.

The time to failure is a random variable, while the Mean time to failure is the expected value, the units of which are time units (e.g., hours). The mean time to failure (MTTF) is defined as follows:

$$
M T T F=H[X]=\int_{0}^{\infty} t \cdot f_{X}(t) d t
$$

Considering that the random variable ' $\mathrm{X}$ ' is exponentially distributed with the failure rate ' $\lambda$ ',

$$
\begin{gathered}
f(t)=\lambda e^{-\lambda t} \\
M T T F=H[X]=\int_{0}^{\infty} t . f(t) d t=\lambda \int_{0}^{\infty} t . e^{-\lambda t}
\end{gathered}
$$

Integrating by parts gives us

$$
M T T F=\frac{1}{\lambda}
$$

MTTF is measured as the likelihood of a system to fail and is represented as units of hours. It is used as a measure to understand the availability and reliability of the systems.

\section{Development of Interdependent Healthcare Critical Infrastructure Network Simulation Model}

The utility of the SCPN based interdependent HCI simulation model during a flooding disaster is shown using a comprehensive case study of a real-world situation. Using the developed system the critical infrastructure failure metric is measured, and the propagation of cascading failures in the Chennai area during the flooding event in December 2015 is evaluated, analyzed, and visualized in a spatiotemporal environment. The real-world case study showcases the dire need of the proposed simulation model system.

\subsection{Healthcare Critical Infrastructure Interdependency during Chennai Flood 2015-Chennai, India}

Chennai is located in the southern part of India and is the capital city of the Tamil Nadu state. It is one of the four major metropolitan cities of India. The city of Chennai and its suburb areas received heavy rainfall events during November-December 2015 that inundated the districts of Chennai, Kanchipuram, and Tiruvallur. Around midnight of 2 December 2015, land in more than $4 \mathrm{~km}$ radius around Adyar River, which flows through the center of Chennai was underwater.

RISAT-1 satellite data of 3 and 4 December 2015 was acquired from the National Remote Sensing Centre (NRSC), Disaster Division, Hyderabad, India. The satellite data was analyzed, and the flood 
inundation layer was generated. Different thematic layers of various CI facilities (example, hospitals, electrical substations, etc.) were overlaid with the flood inundation layer to generate the Chennai flood inundation map. The inundated healthcare facilities map of Chennai city during the Chennai Floods 2015 is shown in Figure 7.

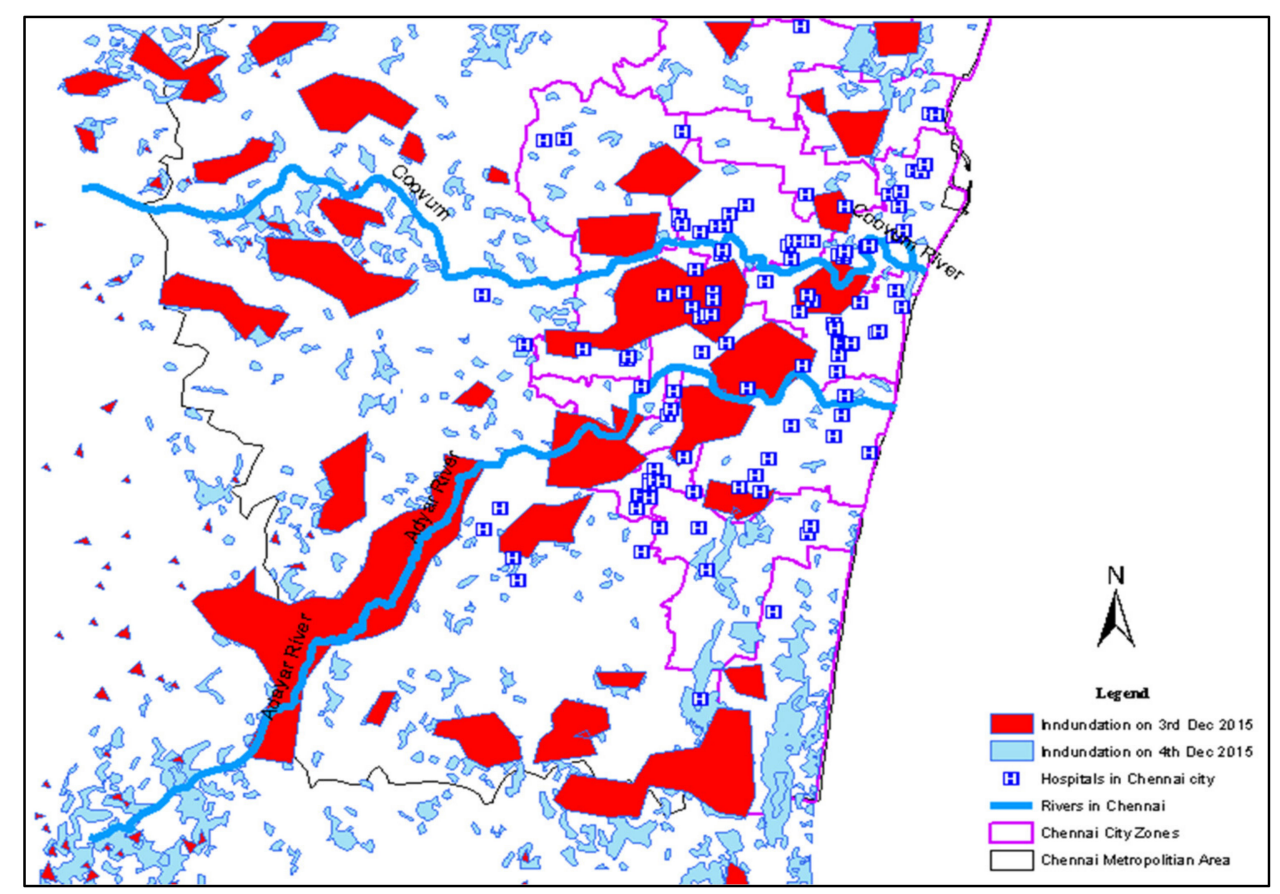

Figure 7. Inundated Healthcare facilities in Chennai during the Chennai Floods 2015. (3 December 2015 and 4 December 2015).

As floodwater levels rose in Chennai, large geographic areas got inundated; the red zones in the map show the substantial inundated regions. Critical infrastructure networks and normal life had come to a halt. Houses, hospitals, roads were flooded, communication networks went down, and there was extensive damage to the electrical power supply. The inundation and flooding of large areas of the Chennai city and suburbs of the adjoining two districts caused disruption of services from existing drinking water supply systems. On account of the inundation of large areas in the city and consequent submergence of the electrical distribution system in floodwater, most of the electrical substations were shutdown. As there was extensive damage to the electrical power infrastructure in Chennai, the electrical supply to the Chennai city was disrupted. Most of the electrical substations reported a rise of floodwater more than 4 feet.

\subsection{Case Study: Interdependent HCI Network during Chennai Floods in 2015}

The case study consists of a health care CI facility (hospital) in Chennai and its interdependent CI services such as electric supply CI (electric substations) and water supply CI (water pumping stations). The hospital is located in Chennai and the name of the geographical area and hospital and its interdependent CI facilities are kept confidential. During the Chennai floods 2015, the operation of the healthcare facility (Hospital $A$ ) and its interdependent $\mathrm{CI}$ also failed due to the increase in the flood level, however as the healthcare facility (Hospital A) was prepared for such adversities, they could evacuate the patients to a safer hospital with the help of boats and ambulances. The simulation model is been used to study another interdependent HCI network during Chennai floods, however, the example is not shown in this article. The following section describes the case study in detail.

Hospital A-Chennai floods 2015: Hospital $A$ is situated in the marshlands of Chennai. The hospital is connected to the main road via a $2 \mathrm{~km}$ arterial road. Due to heavy rains from the 15th of 
November 2015, there was significant flooding of low-lying areas. The hospital was briefly cut off from the main road. Elective procedures got stopped, but the care of the inpatients was not affected. The number of patients admitted to the hospital was under 100 because of elective admissions due to high rainfall. However, on 1 December 2015 news regarding breaching a major lake near the hospital was reported to Hospital $A$. The $2 \mathrm{~km}$ arterial road which connects the hospital to the main road started flooding and in few hours, the hospital was surrounded by 8 feet deep water [45].

The healthcare machinery was placed in the rooms on the ground floor of the hospital. Due to flooding, these rooms were inundated. The Electrical Substation $A$ which supplies electrical supply to Hospital $A$ was submerged in floodwater, due to which the electrical supply to Hospital $A$ got disrupted. However, as the hospital authorities were disaster prepared, they could estimate the CI dependencies and evaluate the response time. The patients were being evacuated from Hospital $A$ with the help of fishing boats and then transported to safer healthcare facilities via ambulances.

\subsection{Assessing the Flood Risk at Individual Critical Infrastructure Facilities}

The uncertainty around the response of critical infrastructure facilities to flood hazard makes it logical to use probabilistic fragility curves. The fragility curves are developed after analyzing the critical infrastructure facility's performance in response to the intensity of the flood hazards experienced. Fragility curves for the CI facility are developed to understand the probability of failure of the CI facility for a flood level. There are many methods to create fragility curves. However, as there are several CI facilities, it is not possible to analytically model each CI facility. Hence empirical data from past flooding events are the main source of information [46,47]. We can develop the fragility curves by analyzing the empirical data from past events, and engineering judgment is viewed as a last resort due to the subjective results it produces [46]. Hence, engineering judgment will only be used where there is no empirical data. And the relationship between the flood hazard and CI facility impact can be easily envisaged (e.g., the depth of water required to close an electrical substation).

\subsubsection{Impact of Flooding on Electrical Supply Critical Infrastructure (Electrical Substation)}

To analyze the impact of flooding on electrical substations, we should have the information about the location of the substation and the height of critical electrical equipment. According to Indian electricity rules the formation level (FL) of an electrical substation has to be fixed at a minimum of $600 \mathrm{~mm}$ higher than the surroundings based on the drainage conditions and the highest flood level in the area [48]. However, the information on failures is less and mainly in the form of the CI facility and news reports. According to the Federal Emergency Management Agency (FEMA) report [47], the functionality threshold depth for an electrical substation is $1.2 \mathrm{~m}$. In a case study referring to electrical substations of a national grid [49], most of the substations had the resilience to flooding to an approximate depth of $0.3 \mathrm{~m}$. By the information, from the experts working in the electrical substations; most of the substations in Chennai would shut down if the flood depth increases more than $0.5 \mathrm{~m}$. The electrical supply CI facilities considered for the case study are as follows: Electrical Substations which supply electricity to Hospital A.

- Electrical Substation A [110/33/11 kV, Date of commissioning: 1 April 1992].

- Electrical Substation B [230/110 kV, Date of commissioning: 13 May 2010].

As the oldest substations commissioned is Electrical Substation A. The flood events, rainfall data after the year 1992, and few assumptions are considered to plot the probability of failure for Electrical Substation A. Table 1 below shows the probability of failure of substation for different flood levels. The fragility curve plot for Electrical Substation A, for various flood levels, is shown in Figure 8. The fragility curve plot shows that the flooding of the substation would start at $0.1 \mathrm{~m}$ and the probability of ' 1 ' indicates the complete shutdown of the substation due to flooding. 
Table 1. Probability of failure of Electrical Substation A for different flood levels.

\begin{tabular}{cc}
\hline Flood Level (m) & Probability of Electrical Substation 'A' (110/11 kV) Flooding \\
\hline 0.1 & 0.333 \\
0.2 & 0.475 \\
0.3 & 0.67 \\
0.4 & 0.84 \\
0.5 & 0.968 \\
0.6 & 1 \\
0.7 & 1 \\
\hline
\end{tabular}

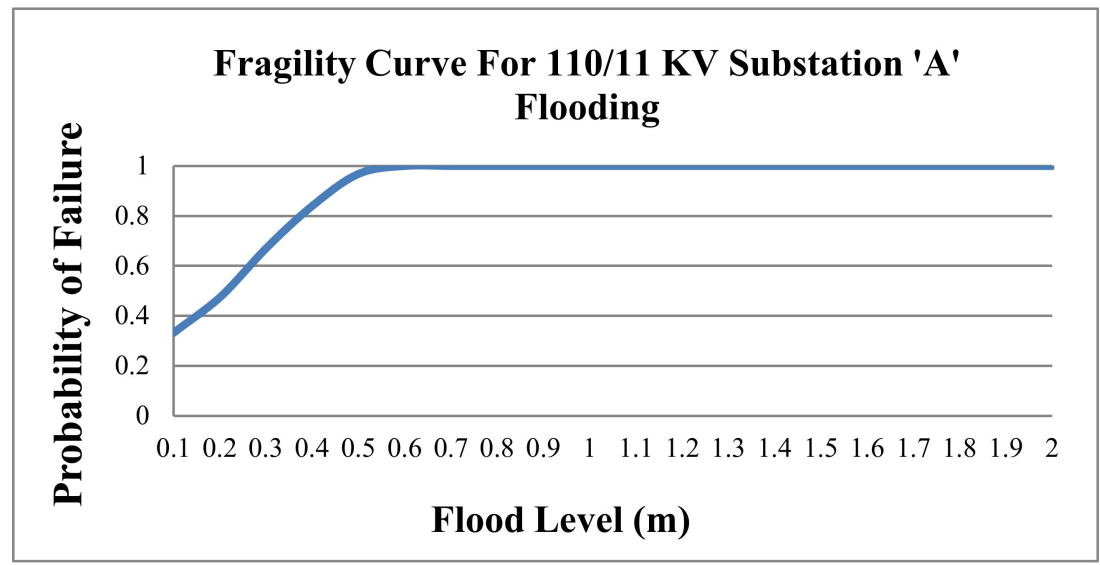

Figure 8. Fragility curve plot for Electrical Substation A (110/11 kV).

\subsubsection{Impact of Flooding on Healthcare Critical Infrastructure (Hospital)}

To analyze the impact of flooding on an HCI facility, data about the location of the HCI facility has to be known. The level of flood inundation level, which can affect the functioning of the healthcare facility, should also be known. As explained earlier in Section 3.2, Hospital $A$ is situated in the marshlands of Chennai, close to a major lake. In 2015 floods Hospital $A$ was surrounded by $8 \mathrm{ft}$ water. During the field visit, it was observed that the hospital is divided into two buildings having two floors each. The hospital equipment on the ground floor was affected due to $2 \mathrm{ft}$ floodwater. Information collected by various sources concludes that Hospital $A$ would get flooded if the flood level is around $0.6 \mathrm{~m}$ approximately to $2 \mathrm{ft}$. An example is given below to calculate the probability of reaching the state of flooding for Hospital $A$.

Number of Occurrence of a flood level of $0.6 \mathrm{~m}$ or more $=20$

Number of times that Hospital ' $\mathrm{B}$ ' has reached the flooding state $=9$

Probability of reaching the state of flooding for Hospital B $=9 / 20=0.45$

\subsubsection{Impact of Flooding on Water Supply Critical Infrastructure (Water Pumping Station)}

To analyze the impact of flooding on water pumping stations, we should know the level of floodwater level, which will cause the pumping station to stop operating. The probability of flooding of the water pumping station at Hospital $A$ is calculated as follows; As the pumping station is present at the same elevation as Hospital $A$, the flood level at which the hospital will get inundated the pumping station would also reach the inundation state at the same elevation.

By analyzing the impact of flooding on each critical infrastructure, one can estimate the probability of failure of a CI facility as explained in Section 2.4. The probability of failure of a CI facility is used for providing the time delay to the transitions in the simulation model. Table 2, showcases the various timed transitions and their corresponding failure rate in our stochastic colored Petri net model. 
Table 2. List of few timed transitions in the interdependent HCI stochastic colored Petri net.

\begin{tabular}{ccc}
\hline Transition & Description & Transition Rate $(\lambda)$ \\
\hline T1 & Occurrence of Flood & Deterministic \\
T9 & The occurrence of flood affects Electrical substation A $(230 / 110 \mathrm{kV})$ & 0.96 \\
T10 & The occurrence of flood affects Electrical substation B $(110 / 11 \mathrm{kV})$ & 0.96 \\
T11 & The occurrence of flood affects Hospital A & 0.45 \\
T8 & The occurrence of flood affects Water pumping station A & 0.45 \\
T10 & Failure in Electrical substation A affects Electrical substation B & 1 \\
T12 & Failure in Electrical substation B affects Water pumping station A & 1 \\
T13 & Failure in Water pumping station A affects Hospital A & 1 \\
\hline
\end{tabular}

\subsection{Stochastic Colored Petri Net (SCPN) Simulation Environment}

This section describes the development of the stochastic colored Petri net model, and how the model was used to evaluate the performance of the interdependent healthcare critical infrastructure network for the Chennai Floods 2015 case study. The impacts of the risk mitigation strategies under different levels of model parameters are observed. As mentioned earlier, the interdependent healthcare CI network-based Stochastic colored Petri net is based on the case study mentioned in Section 3.2. Following are the subnets:

- Flood Simulation.

- $\quad \mathrm{HCI}$ with other CI Interdependency Network.

- HCI Internal Dependencies.

- $\quad$ Status of the Entire Network.

(a) Flood Water Level Simulation Subnet:

As flood level in any area depends on a few variables such as rainfall, soil conditions, wind, temperatures, etc., it is considered as a random variable. It is found that there is an increase in flood levels at random intervals of time. The interdependent CI places nodes in the Petri net-based simulation model represent the states of the different CI facilities such as Electrical substation A, Water Pumping station $A$, Hospital $A$. In our case study, the $\mathrm{CI}$ facilities are located at different geographical locations with different elevations. The $\mathrm{CI}$ facilities are inundated at different flood water levels and at random intervals of time, considering the elevation of the CI facility. For example, Hospital $A$ gets flooded for an elevation of $2 \mathrm{ft}$ at time $\mathrm{t}_{1}$. However, Electrical substation $A$ might get flooded at an elevation of $3 \mathrm{ft}$ at time $\mathrm{t}_{9}$. As mentioned earlier the Petri net simulation model is been divided into four subnets. The flood simulation subnet simulates the flood levels at the various CI facilitates located at different elevations. In the flood simulation subnet, as shown in Figure 9 the flood levels vary upon the intensity of the rainfall and the geographic area, if the rainfall intensity is high then the CI facilities will get inundated at a faster rate than the rainfall at a lower intensity. Considering the Electrical substation $A$ would get flooded at $3 \mathrm{ft}$, as shown in Figure 8. The token from the 'Flood simulation subnet' arrives at 'ES1 place node' indicating the rising flood levels at the Electrical substation A CI facility. The token is fired from the 'ES1 place node' to the 'transition $t_{9}$ '.

The 'transition $t_{9}$ ' is a timed transition, where the token is fired following an exponential time delay function (the rate at which the $\mathrm{CI}$ facility will get flooded). The transition rate is calculated based on the calculations (mentioned in Sections 2.4 and 3.3 earlier). For example, the rate at which the CI facility Electrical substation $A$ will get flooded is 0.64 (the time delay for various transitions has been explained in Table 2 in Section 3.3.3). As the flood level at the Electrical substation A reaches $3 \mathrm{ft}$ level, the 'transition tg' fires a token with a random timestamp to the 'flooded ES1 place node'. The 'flooded ES1 place node' gets enabled, as the token arrives at the place node, indicating the inundation of CI facility Electrical substation A. 


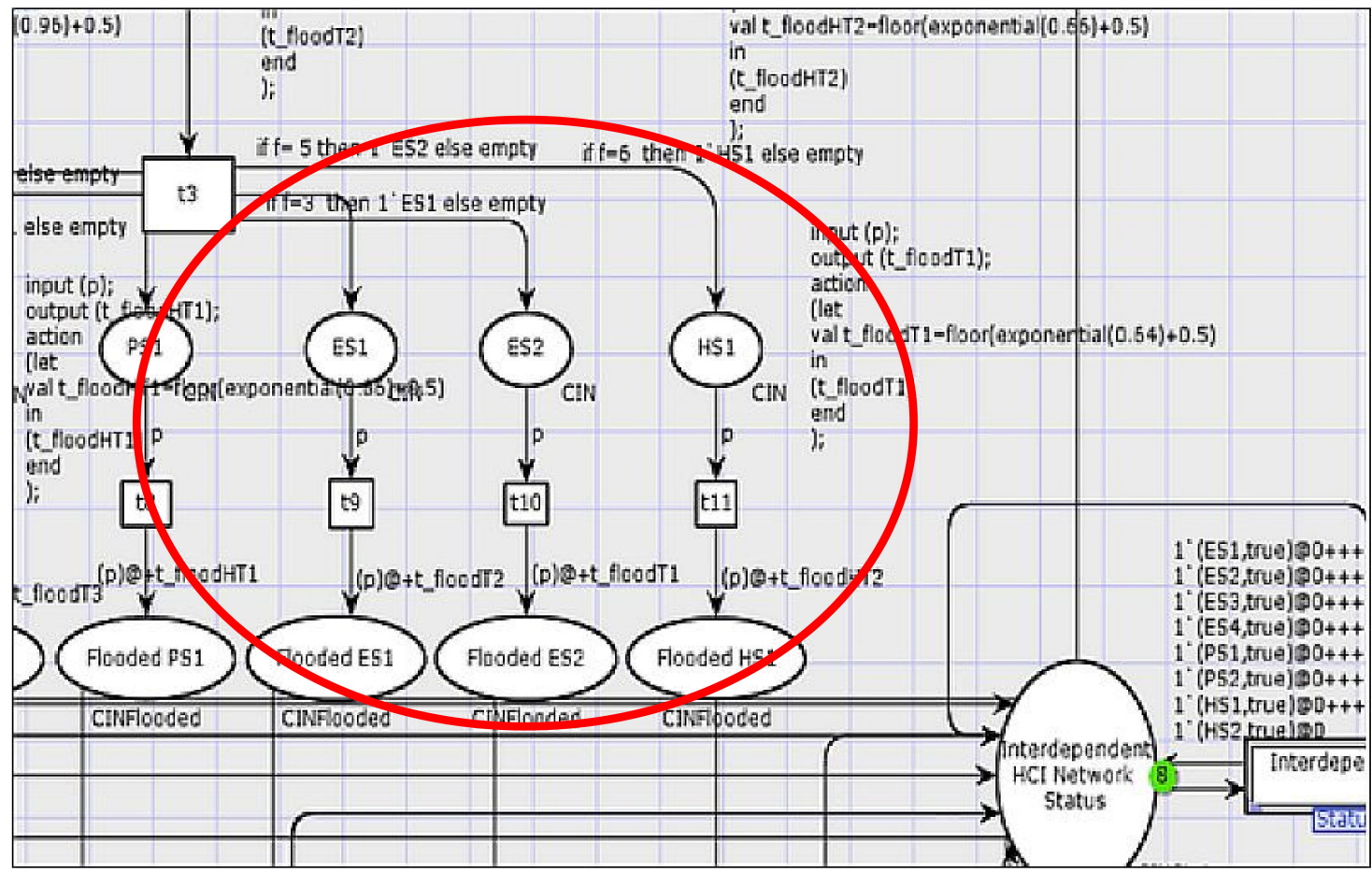

Figure 9. A snippet of the Flood Simulation Subnet.

(b) HCI with other CI Interdependency Network Subnet:

The case study from Section 3.2 is implemented by building a stochastic colored Petri net simulation model, as shown in Figure 10. The HCI with other CI interdependency network subnet is the main subnet where the cascading failure scenario from the case study has been implemented. The various $\mathrm{CI}$ facilities nodes in an interdependent $\mathrm{HCI}$ network considered for the simulation model are the following,

- $\quad$ Electrical Substations $=2$ nodes.

- $\quad$ Hospitals $=2$ Nodes

- Water Pumping station $=1$ node

- $\quad$ Control Room (Command Centre) $=1$ nodes

- $\quad$ Ambulances $=4$

The flood simulation subnet provides the "HCI with other CI interdependency network subnet" with the flooded CI facilities place nodes. Given below is an explanation of the cascading failure simulation of Hospital A and its interdependent CI services form the "HCI with other CI interdependency network subnet" Inundation of the Electrical substation A results in the non-operational state of Electrical substation A.

The non-operational state of Electrical substation A enables the 'transition SF1' to fire a token to 'P4 place node'. The presence of a token enables the ' $P 4$ place node', which represents the state of electrical service disruption to Electrical substation $B$ due to failure in Electrical substation $A$. The impact of the cascading failure of Electrical substation $A$ on Electrical substation $B$ is simulated by firing the token from the 'P4 place node' to 'transition SF2'. For the Electrical substation $B$ to go into a complete non-operational state, the electrical supply form Electrical substation $A$ should be disrupted and the Electrical substation $B$ should be inundated. The Electrical substation $B$ will get inundated when the 'flooded ES2 place node' will get enabled on receiving the token from the 'flood simulation subnet'. As the 'transition SF2' receives tokens from 'P4 place node' and 'flooded ES2 place node', the Electrical substation B reaches a non-operational state. 


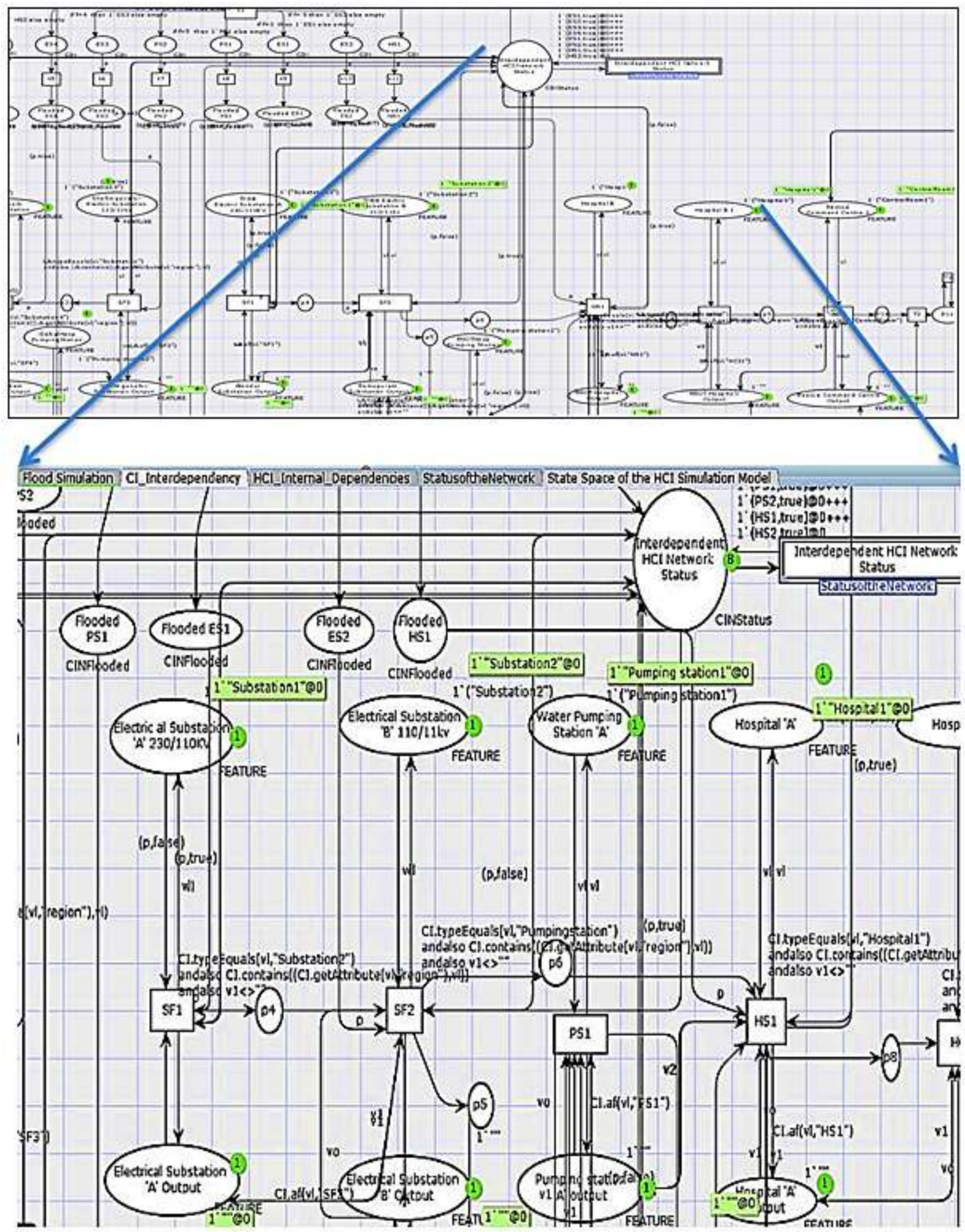

Figure 10. Interdependent healthcare critical infrastructure network colored stochastic Petri net.

The failure of Electrical substation B disrupts the electric supply to the Water Pumping station A and the Hospital $A$. This is simulated by the firing of tokens from the 'transition SF2' to 'place node P5' and 'place node $P 6^{6}$ ' which represent the state of electric supply disrupted to Water Pumping station $A$ and Hospital A respectively. Another token is fired from the 'transition SF2' to the Petri net subnet "Status of the Interdependent HCI network" which indicates the failure of the electrical substation ' $\mathrm{B}$ ' CI facility node in the interdependent HCI network. Similar to the electrical substations' failure, the water 
pumping station arrives at a non-operational state due to a cascading failure scenario. Due to the disruption of electrical and water supply services, Hospital $A$ is affected.

A healthcare CI facility such as Hospital $A$ is not only dependent on external CI services such as electrical supply and water supply but also internal dependencies (explained in Section 3.4 (c)) such as oxygen supply, backup generators, battery supply, food, water, etc.). Hospital $A$ checks for its internal dependencies on the "Internal Dependencies Subnet" of the HCI simulation model. The internal dependencies subnet (explained in Section 3.4 (c)) checks the internal dependencies in the hospital required for the operation of the hospital until the emergency services arrive.

The status results from the "Internal Dependencies Subnet" is been then communicated to the rescue command center requesting evacuation of patients from Hospital A to Hospital C. The command center checks in for the nearest ambulance available and sends it to the impacted hospital for the evacuation of patients. The nearest ambulance reaches the Hospital $A$ for evacuation, choosing the shortest route and sends a message to the command center about reaching its destination. The 'transition HS1' fires another token when enabled to the subnet "Status of the Interdependent HCI network" which indicates the failure of the Electrical Substation A node in the interdependent HCI. Few of the different place nodes in the subnet; the description is given in Table 3.

Table 3. Description of Few place nodes (states) in the Interdependent HCI network SCPN Subnet.

\begin{tabular}{|c|c|c|}
\hline Sr.No & $\begin{array}{l}\text { Name of the State } \\
\text { (Place Node) }\end{array}$ & State (Place Node) Description \\
\hline 1 & Flooded ESI & $\begin{array}{l}\text { The place node indicates the inundation state of the Electrical substation A } \\
\text { due to an increase in the flood level. }\end{array}$ \\
\hline 2 & Flooded ES2 & $\begin{array}{l}\text { The place node indicates the inundation state of the Electrical substation } B \\
\text { due to an increase in the flood level. }\end{array}$ \\
\hline 3 & Flooded PSI & $\begin{array}{l}\text { The place node indicates the inundation state of Water pumping station A, } \\
\text { due to an increase in the flood level. }\end{array}$ \\
\hline 4 & Flooded HSI & $\begin{array}{l}\text { The place node indicates the inundation state of Hospital } A \text {, due to an } \\
\text { increase in the flood level. }\end{array}$ \\
\hline 5 & $\mathrm{P} 4$ & $\begin{array}{l}\text { The place node indicates the non-operational state of the Electrical substation } \\
B \text { (Substation is inundated and electric supply is disrupted). }\end{array}$ \\
\hline 6 & P5 & $\begin{array}{l}\text { The place node indicates the electric supply disruption at Water pumping } \\
\text { station } A \text {, due to the non-operational state of Electric substation B. }\end{array}$ \\
\hline 7 & P6 & $\begin{array}{l}\text { The place node indicates the electric supply disruption at Hospital } A \text {, due to } \\
\text { the non-operational state of Electric substation } B \text {. }\end{array}$ \\
\hline 8 & P7 & $\begin{array}{l}\text { The place node indicates the non-operational state of the Water pumping } \\
\text { station } A \text { (water pumping station is inundated and electric supply is } \\
\text { disrupted). }\end{array}$ \\
\hline 9 & P8 & $\begin{array}{l}\text { The place node indicates the non-operational state of Hospital } A \text {, due to the } \\
\text { inundation of hospital, electric supply, and water supply disruption. }\end{array}$ \\
\hline 10 & $\begin{array}{l}\text { Interdependent } \\
\text { HCI network status }\end{array}$ & $\begin{array}{l}\text { The tokens arriving at the place node indicate the non-operational state of } \\
\text { the various CI facilities with the time stamp. The place node connects to the } \\
\text { HCI network monitoring status subnet. }\end{array}$ \\
\hline
\end{tabular}

(c) HCI Internal Dependencies Subnet:

The operation of a healthcare facility depends not only on the other interconnected critical infrastructure facilities but also on a similar web of internal dependencies. The "HCI internal dependencies subnet" of the simulation model simulates the various internal dependencies of a healthcare facility. Figure 11 shows the subnet of the SCPN, considering the HCI internal dependencies, whereas the description of the place nodes is given in Table 4. 


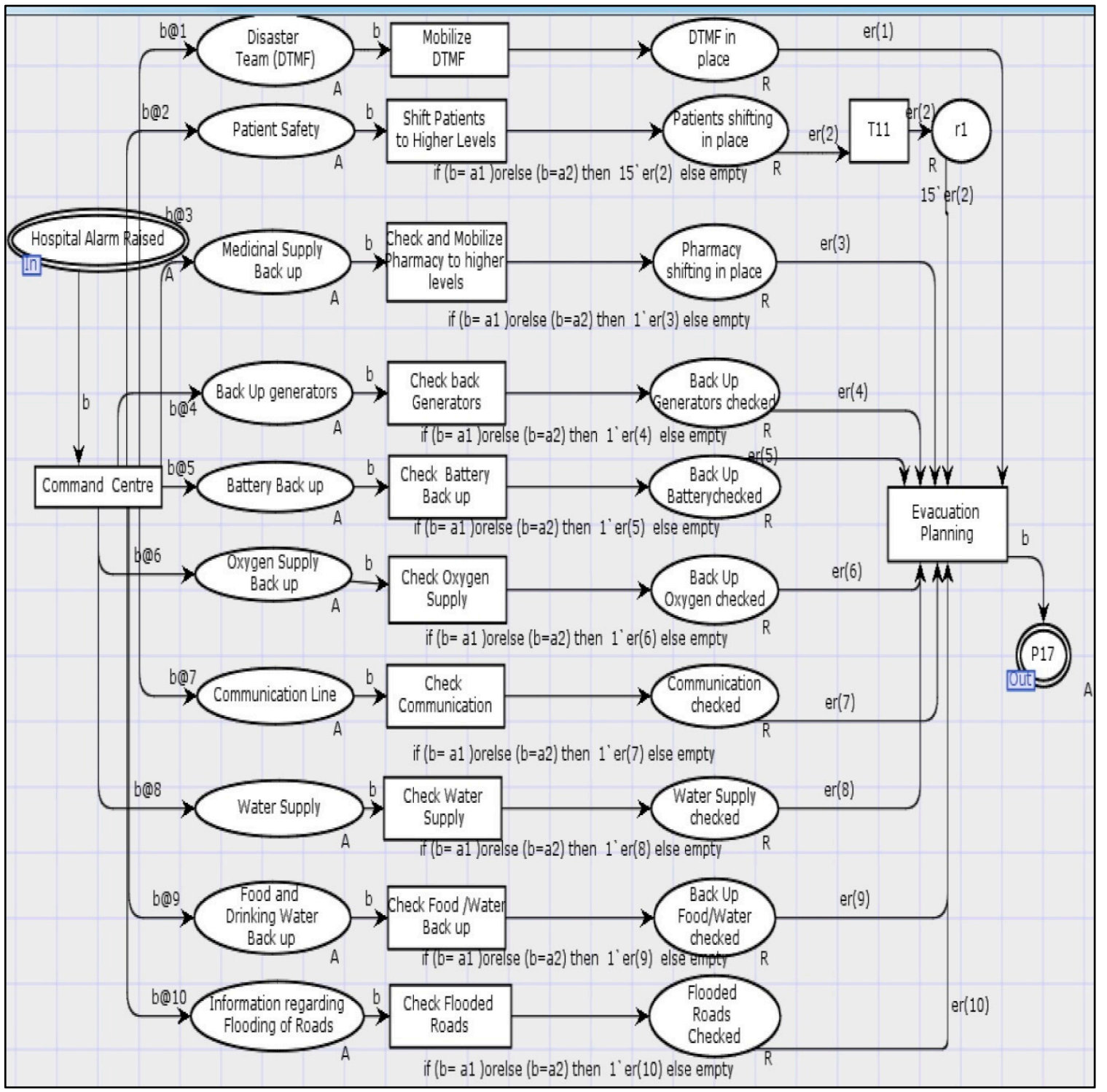

Figure 11. Healthcare critical infrastructure internal interdependencies.

Internal dependencies are related to the operational functionalities inside a healthcare facility such as back up electrical generators, food, and water supply, pharmacy supply, battery backup, oxygen supply, communication, disaster protocol, etc. For example, when an electrical CI facility node (for ex: Electrical Substation A) is flooded, and the electrical supply to the corresponding HCI facility node (for ex: Hospital $A$ ) is disrupted. The electrical backup generators have to start supplying electricity to the healthcare facility. The probability of failure and the time to failure for such generators should also be considered.

The failure of these dependencies requires immediate evacuation of patients to another safer healthcare facility. When the 'transition healthcare $C I$ ' is enabled on the "HCI with other CI interdependency network" subnet is triggered, due to unavailability of electrical CI and water supply CI (for ex: Water Pumping station A) and inundation of the HCI facility (for ex: Hospital A) the "HCI internal dependencies subnet" is triggered. The "HCI internal dependencies" simulation model checks all the internal dependencies and then depending on the status of the severity of emergency at the hospital. The command center helps in evacuation of the patients to a nearby safe healthcare facility (for ex: Hospital C). 
Table 4. Description of few place nodes in the Internal Dependencies of an HCI facility Subnet.

\begin{tabular}{|c|c|c|}
\hline Sr.No & $\begin{array}{l}\text { Name of the State } \\
\text { (Place Node) }\end{array}$ & State (Place Node) Description \\
\hline 1 & Hospital Alarm Raised & $\begin{array}{l}\text { The place node indicates that the alarm state of the hospital, as the } \\
\text { model predicts the time until the hospital might run into a } \\
\text { non-operational state. }\end{array}$ \\
\hline 2 & Patients shifting & $\begin{array}{l}\text { The patients are shifted to secure or higher locations in the hospital, } \\
\text { till emergency evacuation takes place. }\end{array}$ \\
\hline 3 & Pharmacy status & The state indicates the available pharmacy status at the hospital. \\
\hline 4 & $\begin{array}{l}\text { Backup Electrical } \\
\text { Generators status }\end{array}$ & $\begin{array}{l}\text { The place node indicates the status of the backup electrical } \\
\text { generators at the hospital. }\end{array}$ \\
\hline 5 & Power Battery Status & $\begin{array}{l}\text { The place node indicates the status of the Power Battery Status at } \\
\text { the hospital. }\end{array}$ \\
\hline 6 & Oxygen cylinders & $\begin{array}{l}\text { The place node indicates the status of the Oxygen cylinders at the } \\
\text { hospital. }\end{array}$ \\
\hline 7 & Communication & $\begin{array}{l}\text { The place node indicates the status of the communication lines at } \\
\text { the hospital. }\end{array}$ \\
\hline 8 & Food and drinking water & $\begin{array}{l}\text { The place node indicates the current status of the Food and drinking } \\
\text { water to sustain at the hospital, till evacuation. }\end{array}$ \\
\hline 9 & Back up water supply & $\begin{array}{l}\text { The place node indicates the status of the Backup water supply at } \\
\text { the hospital }\end{array}$ \\
\hline 10 & The status of nearby roads & $\begin{array}{l}\text { The place node indicates the status of the nearby roads (Transport } \\
\mathrm{CI}) \text { to the hospital, if they are inundated or not. }\end{array}$ \\
\hline 11 & $\begin{array}{l}\text { Emergency Evacuation } \\
\text { planning }\end{array}$ & $\begin{array}{l}\text { After checking the internal dependencies, the hospital reports about } \\
\text { its status to the command center for emergency evacuation }\end{array}$ \\
\hline
\end{tabular}

(d) Status of the Entire Network Subnet:

The status of the entire network subnet of the simulation model, as shown in Figure 12, monitors the performance of the various interdependent CI nodes. During the simulation as the CI, facility nodes reach to failure state due to the flood inundation and disruption of $\mathrm{CI}$ services. By analyzing the data collected from various simulation runs. We can estimate the meantime to failure of Electrical substation $A$. The interdependent CI nodes fail at different intervals of time. Every CI node will fail at a particular random time for every simulation run. Considering the mean of all the time to failure of a CI node for ' $n$ ' number of simulations will give us the meantime to failure (MTTF) of a CI node. Table 5 describes the $\mathrm{CI}$ facility breakdown states in the $\mathrm{HCI}$ interdependencies status monitoring subnet.

The place nodes "ES1 down" on the "Status of the entire network" subnet indicate the breakdown of Electrical substation A. Similarly when all the CI node failure transitions are triggered, it indicates that the CI nodes are disrupted and are not in operating status. This results in the collapse of the interdependent healthcare critical infrastructure network, for ' $n$ ' number of simulations, one can estimate the meantime to failure of the interdependent healthcare critical infrastructure network. 


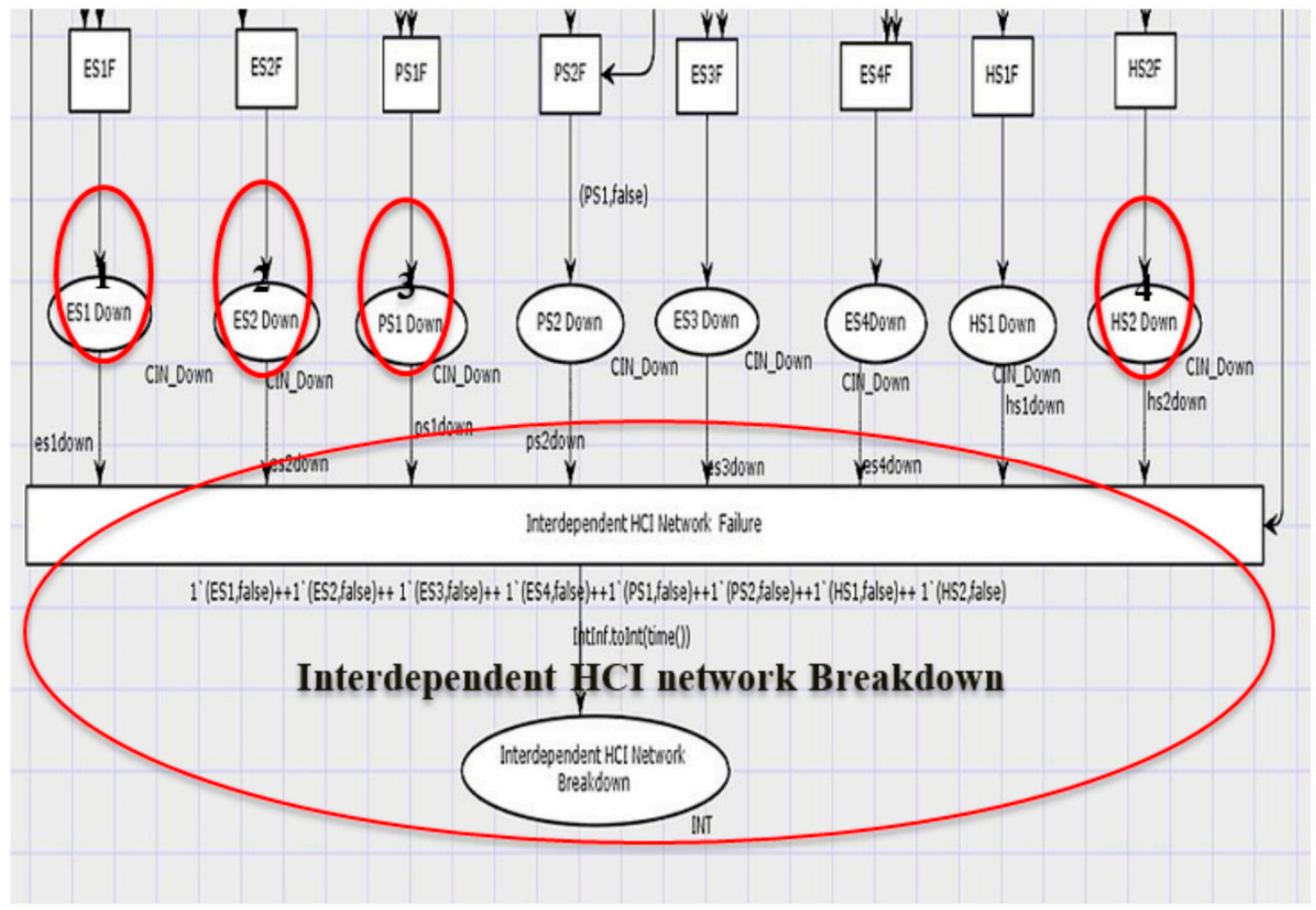

Figure 12. A snippet of HCI interdependencies status monitoring subnet.

Table 5. Description of CI facility breakdown states in the HCI interdependencies status monitoring subnet.

\begin{tabular}{ccc}
\hline Sr.No & $\begin{array}{c}\text { Name of the State } \\
\text { (Place Node) }\end{array}$ & State (Place Node) Description \\
\hline 1 & ESI Down & The place node indicates the complete failure state of the Electrical substation A. \\
2 & ES2 Down & The place node indicates the complete failure state of Electrical substation B. \\
3 & PSI Down & The place node indicates the complete failure state of Water Pumping station A. \\
4 & HSI Down & The place node indicates the complete failure state of Hospital A. \\
\hline
\end{tabular}

\section{Results}

This section presents us with the results of the simulation model. System analysis of the simulation model in Section 4.1 analyzes and evaluates the temporal dimension of the SCPN based HCI simulation model based on the Chennai floods 2015 case study, whereas the spatial dimension is presented in the Section 4.2 SCPN based interdependent HCI simulation model driven geo-visualization interface.

\subsection{System Analysis of the SCPN based Interdependent HCI Simulation Model}

Dependability system evaluation analysis is devoted to investigating the possible manifestations of failures (when and how they can occur) and their impact on the system. The analysis is also used to prevent such failure disruptions and ways to mitigate them. Dependability system analysis provides us with solutions to evaluate the probability and the time of the first occurrence of such a catastrophic condition.

(a) Prediction of Time to Failure of a Critical Infrastructure Facility during a Flood Event

The simulated data is extracted from the states of the model and from the events that occur during the simulations. The extracted data is used to calculate the failure metric of the CI facilities and analyze the behavior of the complex model. During a simulation, the stochastic colored Petri net can contain and generate quantitative information about the performance of a system, such as the meantime to failure (MTTF) of a CI node, the number of tokens at the node place signifying the utilization of the CI node, etc. The data from the different transitions is collected by using a different data collector 
monitor for various $\mathrm{CI}$ nodes. The data collector monitor extracts numerical data from the simulation model and calculates statistics for the data, which is collected for a transition. For example, The ES1 MTTF data collector monitor in the HCI simulation model collects data about the time to failure of the Electrical substation $A$ (ES1) place node for the ' $n$ ' number of simulation runs.

The Electrical substation A (ES1) marking size monitor measures the number of tokens present in the Electrical substation A place node in the Petri net model. The monitor calculates the performance measure such as the average and a maximum number of tokens which are present in the Electrical substation A (ES1) place node during ' $n$ ' simulation. This failure measure would help us to analyze the availability of the critical infrastructure node for service.

The results of the system analysis of the simulation model are calculated for a simulation period of 3 days of rainfall and flooding (a total of $72 \mathrm{~h}$ ). As the simulation model is built to perform simulation indefinitely, a breakpoint monitor is required to stop the simulation. The breakpoint monitor in the Petri net model stops the simulation as per the duration required (in our case study the simulation duration was considered for 3 days (72 h).

The data from different monitors in the CPN Tools are written in different text files and a single folder for one set of simulations. Given below in Table 6 is the meantime to failures of the various CI nodes of the interdependent healthcare critical infrastructure network, for a section of the urban city Chennai during the urban flood of 2015. An example of the results shown in Table 6 is as following; Analyzing the simulation results we see that as the flood levels started increasing, the first failure occurs in Electrical substation A $(230 / 110 \mathrm{kV})$ in 4.5 hours from the onset of the flood.

Table 6. Mean Time to Failure of the various CI nodes of an Interdependent HCI network, during an Urban Flood Chennai Case study.

\begin{tabular}{cc}
\hline Critical Infrastructure Node & Mean Time to Failure (MTTF) (hr) \\
\hline Electrical Substation A (230/110 kV) & 4.5 \\
Electrical Substation B (110/11 kV) & 7.4 \\
Water Pumping station A & 8.20 \\
Hospital A & 8.70 \\
\hline
\end{tabular}

Due to the failure of Electrical substation A and increasing flood levels Electrical substation $B$ $(110 / 11 \mathrm{kV})$ is affected. The electric supply to Hospital A provided from Electrical substation $B$ will get disrupted in $3 \mathrm{~h}$, as it would take around $7.5 \mathrm{~h}$ for Electrical substation $B$ to reach a complete non-operational state from the onset of the flood.

The Water Pumping station A is geographically located closer to Hospital $A$ and due to the shutdown of Electrical substation $B$, the electric supply to the water pumping station is disrupted. The estimated time for the complete non-operational state of Water Pumping station $A$ is $8.20 \mathrm{~h}$ from the onset of flooding. This shows that the water supply CI facility reaches a failure state an hour after the failure of the Electrical substation B. From the simulation results, we can infer that show that the time for Hospital $A$ to reach a non-operational state is around $8.7 \mathrm{~h}$ from the onset of the flood. There is not much variation for the time taken to reach a non-operational state between Hospital $A$ and the Water Pumping station A. This is because both Hospital A and the Water Pumping station A are located in the same geographic area. As the hospital gets inundated, from the simulation results we can infer that the hospital authorities can estimate that they have $3 \mathrm{~h}$ (approx.) for electric supply disruption, and around half an hour left for the water supply disruption. The estimation of the time frame can help the hospital authorities to arrange emergency services for the evacuation of patients and disaster prepare the hospital with the backup resources available.

The simulation model is integrated with the geographic information system, and a log window keeps on updating the status of the CI facilities if they are in an operating, inundation state, or a complete failure state. When the electric supply and water supply get disrupted to either of the hospital CI facilities, the hospital CI facilities check for the internal dependencies such as back up 
electrical generator, battery backup, oxygen cylinders, etc. The hospital CI facilities then request the command center for the evacuation of patients. The command center alerts the ambulance services as the hospital is reaching a failure state for the evacuation of patients. The GIS system (explained in the next Section 4.2) shows the evacuation route to a safer hospital and the same is been updated on the $\log$ window.

(b) State Space Analysis Queries for the Simulation Model

The simulation model is quite complex, as it enters into many states. The state-space for the simulation model is vast. However, we can query from the state space graph about the different states of the entire simulation model. For example, if the user wants to know the minimum path from the state where the Electrical substation $A$ has inundated to the state where the entire interdependent HCI network has failed. For example, if the state where Electrical substation $A$ is inundated is node 16 and the entire $\mathrm{HCI}$ network failure state node is 89 the minimum path is: $[16 ; 25 ; 56 ; 76,89]$. From the simulation runs we see that the failure in the electric supply CI network will increase the vulnerability of water supply CI networks. The failure of both electric supply CI and water supply CI will have a perilous impact on the HCI network. The performance simulations show that as the number of interdependencies in an interdependent $\mathrm{HCI}$ network increases, the more vulnerable they are.

\subsection{SCPN Driven Geographic Information System}

A CI facility such as a hospital, electrical substation, road, etc., in the real world, is mapped into a Geographic Information System (GIS) as a critical infrastructure geo-entity. The CI geo-entity is characterized by the type of critical infrastructure facility (such as a hospital, electric substation, water pumping station, a road segment, etc.), the geographic attributes (for example; name, represented by a polygon, point, or a line, location coordinates, etc.). The geographic attributes of a CI geo-entity are important, as the attributes help in linking the CI geo-entity to a unique spatial location and context of its environment. For example, a hospital CI geo-entity is represented as a point feature with its geographical and other attributes such as the location of the hospital, type of hospital, the capacity of beds, etc. A network of such hospital CI geo-entities would represent a thematic dataset of the hospital network.

In a stochastic colored Petri net driven geographic information system, spatial data layers related to the different $\mathrm{CI}$ networks such as electrical supply CI network (the geolocation of electrical substation), water supply CI network (the geolocation of water pumping station, geographic area of water bodies) and healthcare CI network (the geolocation of healthcare facilities) are combined for visual exploration of complex datasets. A JAVA (www.oracle.com/java) code is developed by using CPN tools (www.cpntools.org); Simulator Extensions Java library and Geo-Tools (www.geotools.org), which is an open-source Java, for an SCPN driven GIS simulation model. The interdependent SCPN simulation model communicates with the GIS system using the TCP/IP protocol, which is an implementation of the remote procedure call (RPC) system. Figure 13 showcases the dynamic data exchange between the SCPN simulation model and the geographic information system.

The stochastic interdependencies in the SCPN model are based on various geographical and logical based interdependency rules. Object-oriented based geospatial functions are developed such that the place nodes in the SCPN model are spatially registered, with the CI geo-entities, and the geolocation is color-coded.

The entire SCPN driven GIS model is encapsulated in a JAVA based client interface for Geovisualization as shown in Figure 14, various geospatial-based interactive components are added on the client interface such as zoom in zoom out. Dynamically add notes and markers, switching between different map layers and pan to control the display within its context, can be used by the end-users. 


\section{Stochastic Coloured Petri Net Model}

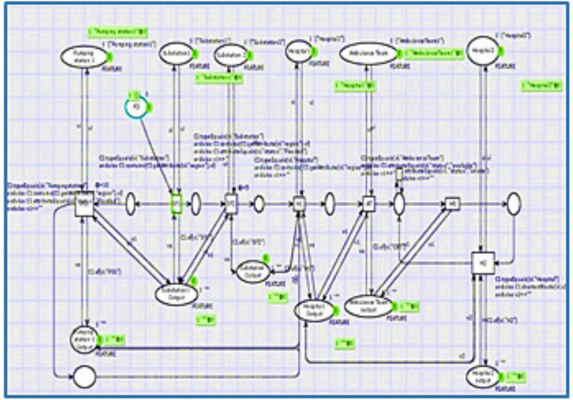

Temporal Dynamics

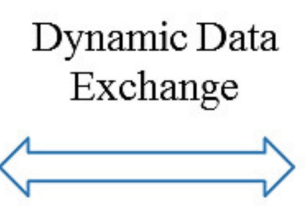

Figure 13. Stochastic Colored Petri net model-driven Geographic Information System.

The guard functions of the transition in the SCPN simulation model are based on the geospatial relation function. These guard functions act like a constraint; once the transition is enabled and the token is fired, the transition returns a Boolean result. Given below in Figure 15 is an example of a transition 'HS1' and a guard function assigned to it. The transition 'HS1' will get enabled to fire a token only when all the constraints are met. The transition first checks the CI geo entity, if it is Hospital A. When the first constraint is met, then the transition checks the region (polygon) in which the hospital A is located in. Then it would check the condition of the region if it is flooded or not. Based on the query and when the transition is fired, the geographic region on the GIS client interface is highlighted by changing the color scheme indicating the geographic region where Hospital A is situated in is "Flooded".

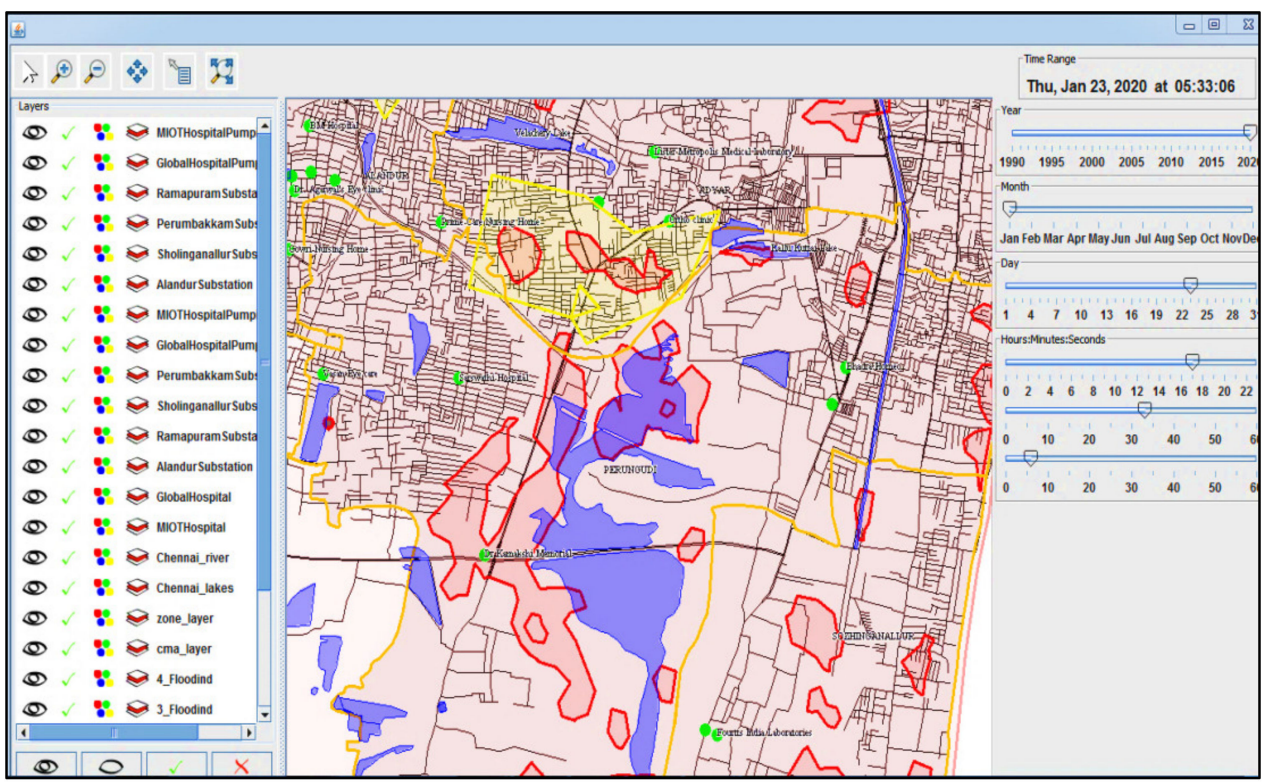

Figure 14. Geo-visualization client interface For Interdependent HCI Petri Net Simulation Model Chennai Case Study.

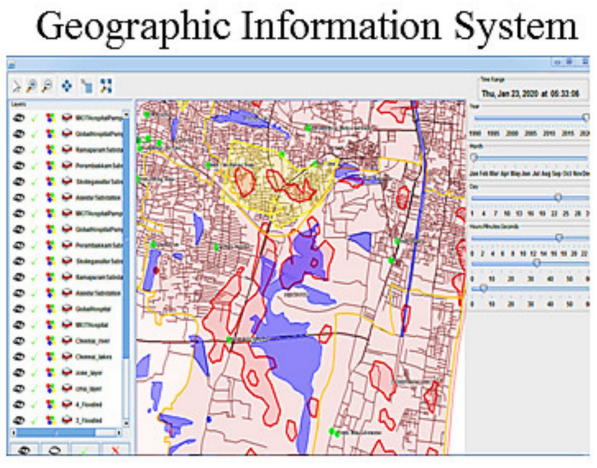

Spatial Dynamics 


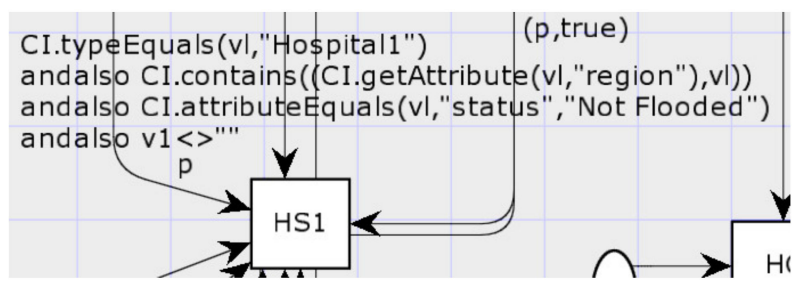

Figure 15. Transition HS1 in the SCPN model with geographical based constraints.

Various queries form the interdependent HCI SCPN simulation model can be queried with the help of different geometric, feature type, and attribute functions. The SCPN driven GIS simulation model can perform various GIS-based analyses such as attribute-based analysis, node-based analysis, spatial area-based analysis, and network-based analysis.

(a) SCPN driven GIS-based Attribute Analysis for Interdependent HCI Network

The spatial knowledge related to CI facilities in the Interdependent HCI CI network is queried from the CI spatial databases. The various place nodes in the Interdependent HCI SCPN simulation model are registered with CI spatial databases. The geospatial relationships based on the geometry of geo-entities and the geospatial based object-oriented functions in the Petri net provides the HCI simulation model with the capability of attribute analysis. The simulation model can query the CI related geographic attribute information (For ex: CI facility such as hospital location, electrical substation location, water pumping station location, etc.). A geodatabase related functions provide the end-user with attribute information about a specific CI facility in a certain geographic location.

(b) SCPN Driven GIS-based Node Analysis for Interdependent HCI Network

The geospatial functions related to the distance between two nodes provide us with information about the distance between two different $\mathrm{CI}$ facilities, for example; the functions display the distance between a hospital A and hospital B for the evacuation of patients. The node (point) based analysis can provide information about the attribute of the point feature, for example for node hospital A, the node base attributes provide information about the type of hospital, the capacity of the hospital, etc. As the place nodes are spatially registered, with the CI facilities, and the geolocation is color-coded. The change in the place node marking in the Petri net model would result in the change in the color scheme of the CI data objects on the GIS-based client interface. For example; The color scheme associated with the point data object registered with a hospital CI facility is blue. At this instant, there is no token present in the hospital CI place node in the Petri net model. However, when the place node is enabled with a token, the color scheme of the point data object registered with a hospital CI facility changes to red. During the simulation run, as a result of a transition being enabled, and a token is fired the SCPN simulation model state changes. As the state changes in the underlying SCPN simulation model, the behavior of the CI geo entity on the GIS-based client interface also changes.

(c) SCPN driven GIS-Based Area Analysis for Interdependent HCI Network

The Area-based analysis provides functions for polygon features. The functions are based on the geometry of the features. The functions are used to provide information about the polygon features related to critical infrastructures. GIS-based operations such as buffer can be used to identify a buffer zone of an HCI facility.

(d) SCPN Driven GIS-Based Network Analysis for Interdependent HCI Network

The transport CI geodatabase consists of nodes and links. Network operation such as proximity analysis is used to analyze the distance between the different CI nodes. Optimal route-finding operations help in determining the shortest path between two nodes (ex: healthcare facilities). This is helpful while evacuating the patients and the ambulance tries to determine the optimal path between the two healthcare facilities. Figure 16 shows the process of evacuation of patients by ambulances from Hospital A to Hospital B. When the simulation model predicts that Hospital A would reach a non-operational state in few hours from system analysis, the command center looks out for available ambulances. We see in the figure that the Ambulanceteam1 is available; the Ambulance team1 is 
services required by society and during an extreme event the need for these services increases. The challenge of cascading failures in the interdependent HCI network is addressed in an Indian scenario. The utility of the approach is shown using a real-world case study, the dynamic performance of the interdependent healthcare critical infrastructure network and propagation of cascading failures in the Chennai area during the Chennai floods 2015 is evaluated and analyzed. The results of the system analysis of the simulation model are calculated for a simulation period of 3 days of rainfall and flooding (a total of $72 \mathrm{~h}$ ). From the simulation runs we see that the failure in the electric supply CI network will increase the vulnerability of water supply CI networks. And the failure of both electric supply CI and water supply $\mathrm{CI}$ will have a perilous impact on the HCI network. From the system analysis, we can derive that the more interdependent the critical infrastructures are, the more vulnerable they are.

The simulation model will help the disaster response personnel or organizations who are managing crisis activities (e.g., Health care managers, Emergency management personnel, etc.). This work addressed this critical area to enable better preparation and management during a disaster. For instance, if the estimate from the proposed simulation model is performed, it gives insights into disruptions in electrical $\mathrm{CI}$, and water supply $\mathrm{CI}$ and how it will impact the healthcare $\mathrm{CI}$ in say seven hours from now. Consequently, a hospital can plan the evacuation of critically ill patients and be better disaster prepared, thus significantly lowering the critical eventualities. The developed model can have an impact on the decision-making heuristics during a flood event. This kind of dynamic real-time understanding of the propagation of cascading failures of CI in an Interdependent CI network simulation would be a crucial component in any disaster response activities.

Author Contributions: Conceptualization, Nivedita Nukavarapu and Surya Durbha.; methodology, Nivedita Nukavarapu and Surya Durbha.; software, Nivedita Nukavarapu; validation, Nivedita Nukavarapu.; formal analysis, Nivedita Nukavarapu.; investigation, Nivedita Nukavarapu.; resources, Nivedita Nukavarapu and Surya Durbha.; data curation, Nivedita Nukavarapu.; writing — original draft preparation, Nivedita Nukavarapu.; writing-review and editing, Surya Durbha.; visualization, Nivedita Nukavarapu.; supervision, Surya Durbha.; project administration, Surya Durbha.; funding acquisition, Surya Durbha. All authors have read and agreed to the published version of the manuscript.

Funding: This research received no external funding.

Acknowledgments: The authors would like to thank the support from the Indian Institute of Technology Bombay and Disaster Division, National Remote Sensing Center (NRSC), Hyderabad, India.

Conflicts of Interest: The authors declare no conflict of interest.

\section{References}

1. Ramachandra, T.; Mujumdar, P.P. Urban Floods: Case Study of Bangalore. J. Disaster Dev. 2009, 3, 1-99.

2. Rinaldi, S.M.; Peerenboom, J.P.; Kelly, T.K. Identifying, understanding, and analyzing critical infrastructure interdependencies. IEEE Control Syst. Mag. 2001, 21, 11-25.

3. Dudenhoeffer, D.; Hartley, S.; Permann, M.; Pederson, P. Critical Infrastructure Interdependency Modeling: A Survey of Critical Infrastructure Interdependency Modeling; Idaho National Laboratory: Idaho Falls, ID, USA, 2006; Volume 25, p. 27.

4. Ouyang, M. Review on modeling and simulation of interdependent critical infrastructure systems. Reliab. Eng. Syst. Saf. 2014, 121, 43-60. [CrossRef]

5. Petit, F.; Verner, D.; Brannegan, D.; Buehring, W.; Dickinson, D. Analysis of Critical Infrastructure Dependencies and Interdependencies; Office of Scientific and Technical Information (OSTI): Oak Ridge, TN, USA, 2015.

6. Vespignani, A. Complex networks: The fragility of interdependency. Nature 2010, 464, 984-985. [CrossRef] [PubMed]

7. Nukavarapu, N.; Durbha, S. Stochastic coloured petrinet based healthcare infrastructure interdependency model. In Proceedings of the International Archives of the Photogrammetry, Remote Sensing and Spatial Information Sciences-ISPRS Archives; ISPRS Congress, Prague, Czech Republic, 12-19 July 2016.

8. Loosemore, M.; Chow, V.W.; McGeorge, D. Modelling the risks of extreme weather events for Australasian hospital infrastructure using rich picture diagrams. Constr. Manag. Econ. 2012, 1-16. [CrossRef] 
9. Lee, E.E.; Mitchell, J.E.; Wallace, W.A. Restoration of services in interdependent infrastructure systems: A network flows approach. IEEE Trans. Syst. Man Cybern. Part C Appl. Rev. 2007, 37, 1303-1317. [CrossRef]

10. Voeller, J.G.; Lee, E.E.; Mitchell, J.E.; Wallace, W.A. Network Flow Approaches for Analyzing and Managing Disruptions to Interdependent Infrastructure Systems. In Wiley Handbook of Science and Technology for Homeland Security; John Wiley \& Sons: Hoboken, NJ, USA, 2009.

11. Chou, C.-C.; Tseng, S.-M. Collection and Analysis of Critical Infrastructure Interdependency Relationships. J. Comput. Civ. Eng. 2010, 24, 539-547. [CrossRef]

12. Jha, M.K. Dynamic Bayesian Network for Predicting the Likelihood of a Terrorist Attack at Critical Transportation Infrastructure Facilities. J. Infrastruct. Syst. 2009, 15, 31-39. [CrossRef]

13. Barrett, C.; Beckman, R.; Channakeshava, K.; Huang, F.; Kumar, V.A.; Marathe, A.; Marathe, M.V.; Pei, G. Cascading failures in multiple infrastructures: From transportation to communication network. In Proceedings of the 2010 5th International Conference on Critical Infrastructure, CRIS 2010, Beijing, China, 20-22 September 2010.

14. Dueñas-Osorio, L.; Kwasinski, A. Quantification of lifeline system interdependencies after the 27 February $2010 \mathrm{Mw} 8.8$ offshore Maule, Chile, earthquake. Earthq. Spectra 2012, 28, 581-603. [CrossRef]

15. Cardellini, V.; Casalicchio, E.; Galli, E. Agent-based modeling of interdependencies in critical infrastructures through UML. In Proceedings of the SpringSim '07-Proceedings of the 2007 Spring Simulation Multiconference, Norfolk, VA, USA, 25-29 March 2007.

16. Arboleda, C.A.; Abraham, D.M.; Richard, J.-P.P.; Lubitz, R. Vulnerability Assessment of Health Care Facilities during Disaster Events. J. Infrastruct. Syst. 2009, 15, 149-161. [CrossRef]

17. Arboleda, C.A.; Abraham, D.M.; Lubitz, R. Simulation As a Tool to Assess the Vulnerability of the Operation of a Health Care Facility. J. Perform. Constr. Facil. 2007, 21, 302-312. [CrossRef]

18. John, J. Healthcare Impact Simulation Using HCSim Energy; U.S. Department of Energy Office of Scientific and Technical Information: Oak Ridge, TN, USA, 2013.

19. Vugrin, E.D.; Verzi, S.J.; Finley, P.D.; Turnquist, M.A.; Griffin, A.R.; Ricci, K.A.; Wyte-Lake, T. Modeling Hospitals' Adaptive Capacity during a Loss of Infrastructure Services. J. Healthc. Eng. 2015, 6, 85-120. [CrossRef] [PubMed]

20. Petri, C.A. Kommunikation mit Automaten. Ph.D. Thesis, University of Bonn, Bonn, Germany, 1962.

21. Peterson, J.L. Petri Nets. ACM Comput. Surv. 1977, 9, 223-252. [CrossRef]

22. Marsan, M.A. Stochastic Petri nets: An elementary introduction. Appl. Evol. Comput. 1990, 424, 1-29.

23. Zuberek, W.M. Timed Petri nets and preliminary performance evaluation. In Proceedings of the 7th annual symposium on Computer Architecture, La Baule, France, 6-8 May 1980; pp. 88-96.

24. Marsan, M.A.; Balbo, G.; Conte, G.; Donatelli, S.; Franceschinis, G. Modelling with Generalized Stochastic Petri Nets. ACM SIGMETRICS Perform. Eval. Rev. 1998, 26, 2. [CrossRef]

25. Gursesli, O.; Desrochers, A.A. Modeling infrastructure interdependencies using Petri nets. In Proceedings of the 2003 IEEE International Conference on Systems, Man and Cybernetics. Conference Theme-System Security and Assurance (Cat. No.03CH37483), Washington, DC, USA, 8 October 2004.

26. Sultana, S.; Chen, Z. Modeling flood induced interdependencies among hydroelectricity generating infrastructures. J. Environ. Manage. 2009, 90, 3272-3282. [CrossRef] [PubMed]

27. Krings, A.; Oman, P. A simple GSPN for modelling common mode failures in critical infrastructures. In Proceedings of the 36th Annual Hawaii International Conference on System Sciences, HICSS 2003, Big Island, HI, USA, 6-9 January 2003.

28. Chen, T.M.; Sanchez-Aarnoutse, J.C.; Buford, J. Petri net modeling of cyber-physical attacks on smart grid. IEEE Trans. Smart Grid 2011, 2, 741-749. [CrossRef]

29. Laprie, J.-C.; Kanoun, K.; Kaâniche, M. Modelling Interdependencies Between the Electricity and Information Infrastructures. Appl. Evol. Comput. 2007, 4680, 54-67.

30. Omidvar, B.; Malekshah, M.H.; Omidvar, H. Failure risk assessment of interdependent infrastructures against earthquake, a Petri net approach: Case study-power and water distribution networks. Nat. Hazards 2013, 71, 1971-1993. [CrossRef]

31. Giglio, D.; Sacco, N. A Petri net model for analysis, optimisation, and control of railway networks and train schedules. In Proceedings of the 2016 IEEE 19th International Conference on Intelligent Transportation Systems (ITSC), Rio de Janeiro, Brazil, 1-4 November 2016. 
32. Di Febbraro, A.; Giglio, D.; Sacco, N. On analyzing the vulnerabilities of a railway network with Petri nets. Transp. Res. Procedia 2017, 27, 553-560. [CrossRef]

33. Szpyrka, M.; Jasiul, B. Evaluation of cyber security and modelling of risk propagation with Petri nets. Symmetry 2017, 9, 32. [CrossRef]

34. Dotoli, M.; Fanti, M.P.; Iacobellis, G.; Martino, L.; Moretti, A.M.; Ukovich, W. Modeling and management of a hospital department via petri nets. In Proceedings of the 2010 IEEE Workshop on Health Care Management, WHCM, Venice, Italy, 18-20 February 2010.

35. Hamana, S.; Augusto, V.; Xie, X.; Ieee, F. A Timed Petri Net Approach for Verification of Territorial Healthcare Information Systems. In Proceedings of the 2016 IEEE International Conference on Automation Science and Engineering (CASE), Fort Worth, TX, USA, 21-25 August 2016.

36. Murata, T. Petri Nets: Properties, Analysis and Applications. Proc. IEEE 1989, 77, 541-580. [CrossRef]

37. Peterson, J.L. A note on colored Petri nets. Inf. Process. Lett. 1980, 11, 40-43. [CrossRef]

38. Jensen, K.; Kristensen, L.M. Coloured Petri Nets: Modelling and Validation of Concurrent Systems; Springer: Berlin/Heidelberg, Germany, 2009.

39. Wells, L. Performance analysis using CPN tools. In Proceedings of the 1st International Conference on Performance Evaluation Methodolgies Tools-Valuetools '06, Pisa, Italy, 11-13 October 2006; p. 59.

40. Jensen, K. In Proceedings of the Sixth Workshop and Tutorial on Practical Use of Coloured Petri Nets and the CPN Tools, Aarhus, Denmark, 24-26 October 2005; p. 300.

41. Jensen, K. Coloured petri nets. In Lecture Notes in Computer Science (Including Subseries Lecture Notes in Artificial Intelligence and Lecture Notes in Bioinformatics; Springer: Berlin/Heidelberg, Germany, 1987.

42. Jensen, K. Coloured Petri Nets: Basic Concepts, Analysis Methods and Practical Use; Springer: Berlin/Heidelberg, Germany, 1996.

43. Van der Aalst, W.M.P.; Stahl, C.; Westergaard, M. Strategies for modeling complex processes using colored Petri nets. In Lecture Notes in Computer Science (Including Subseries Lecture Notes in Artificial Intelligence and Lecture Notes in Bioinformatics; Springer: Berlin/Heidelberg, Germany, 2013.

44. Malhotra, M.; Trivedi, K.S. Dependability Modeling Using Petri-Nets. IEEE Trans. Reliab. 1995, 44, 428-440. [CrossRef]

45. Pandey, S.; Kaliamoorthy, I.; Reddy, M.S.; Rajakumar, A.; Pillai, B.; Micheal, J.C.J.; Kancherla, R.; Rela, M.; Varghese, J. Safe emergency evacuation of a Tertiary Care Hospital during the 'once in a century' floods in Chennai, India. Indian J. Crit. Care Med. 2016, 20, 104-108. [CrossRef]

46. Sill, B.B.L.; Kozlowskf, R.T. Analysis of Storm-Damage Factors for Low-Rise Structures. J. Perform. Constr. Facil. 1997, 11, 168-177. [CrossRef]

47. Federal Emergency Management Agency. Seismic Performance Assessment of Buildings; FEMA P-58. vol. 1, no. September; Federal Emergency Management Agency: Washington, DC, USA, 2012; p. 278.

48. Precautions, G.S. Indian Electricity Rules, 1956; Government of India, Ministry of Power, Central Electricity Board: New Delhi, India, 1956.

49. Peace, J.; Seidel, S.; Peace, J. Business Weathering The Storm: Building Business Resilience Building Business Resilience; Center for Climate and Energy Solutions: Arlington, VA, USA, 2013.

(C) 2020 by the authors. Licensee MDPI, Basel, Switzerland. This article is an open access article distributed under the terms and conditions of the Creative Commons Attribution (CC BY) license (http://creativecommons.org/licenses/by/4.0/). 